\title{
Wireless-Powered Edge Computing with Cooperative UAV: Task, Time Scheduling and Trajectory Design
}

\author{
Xiaoyan Hu, Student Member, IEEE, Kai-Kit Wong, Fellow, IEEE, \\ and Yangyang Zhang
}

\begin{abstract}
A wireless-powered mobile edge computing (MEC) architecture with the cooperation between an access point (AP) and an unmanned aerial vehicle (UAV) is studied in this paper. The AP, powered by the grid, is integrated with a high-performance processing server to help compute the user equipments' (UEs) offloaded tasks while also performing high-power laser-like energy charging for the UAV. The UAV serves as (1) an information relay to help the UEs offload/download their computation tasks/results, (2) an energy relay to broadcast energy from the AP to the UEs, as well as (3) an MEC server to help the UEs compute their tasks. We aim at maximizing the weighted sum completed task-input bits (WSCTB) of UEs under the task and time allocation, information-causality, energy-causality, and the UAV's trajectory constraints, by jointly optimizing the task and time allocation as well as the UAV's energy transmit power and trajectory. The formulated WSCTB maximization problem is non-convex, and we propose a three-step block coordinate descending algorithm to address three sub-problems iteratively for obtaining a proper solution. Simulation results show that the UAV's trajectories highly depend on the AP's location and the UEs' weight values. In addition, significant performance improvement is achieved by the proposed algorithm compared to some practical benchmarks.
\end{abstract}

\section{A. Motivation and Prior Work}

\section{INTRODUCTION}

The upcoming 5G wireless communication technologies and the rapidly growing Internet-ofthings (IoT) result in the explosive growth in the number of mobile and intelligent devices. New mobile applications are also transforming people's life, such as self-driving cars, smart home, virtual and augmented reality, and etc. All of these applications demand a large amount of computing resources, and some may exceed the capability of resource-limited user equipments

The work is supported in part by EPSRC under grant EP/N008219/1.

$\mathrm{X}$. Hu and K.-K. Wong are with the Department of Electronic and Electrical Engineering, University College London, London WC1E 7JE, UK (Email: \{xiaoyan.hu.16, kai-kit.wong\}@ucl.ac.uk). Y. Zhang is with Kuang-Chi Institute of Advanced Technology, Shenzhen, China (Email: yangyang.zhang@kuang-chi.org). This paper was presented in part at the 20th IEEE International Workshop on Signal Processing Advances in Wireless Communications (SPAWC), Cannes, France, Jul. 2019 [1]. 
(UEs). Recently, mobile edge computing (MEC) has emerged as a promising way to deal with this issue [2-4]. The idea is to shift the cloud computing capability to the edge of the communication networks. In so doing, UEs' computation tasks can be offloaded and completed at the edge, e.g., the access points (APs), and even less abled UEs can enjoy low-latency services.

MEC, as a means to improve computing performance, has been actively investigated in recent years [5-10]. Resource allocation for a multiuser MEC system was considered in [6], where the weighted sum energy consumption of UEs was minimized using an offloading priority function. In [7], an energy-aware offloading scheme was proposed to tradeoff between users' energy consumption and the execution latency. Later in [8], joint task offloading and resource allocation was considered to maximize the task offloading gain. A two-tier heterogeneous cloud computing network was studied in [9], where the benefits of coexisting edge and central clouds were investigated. A blockchain based platform was considered for video streaming with MEC in [10], and an incentive mechanism was proposed to facilitate the cooperation of different nodes.

Though MEC lifts the computation capability of UEs, the energy of UEs can greatly limit the overall performance. To address this, [11-14] introduced energy harvesting and wireless power transfer (WPT) into MEC systems, to ensure that sustainable energy is available at the UEs. In [11], WPT was used to maximize the computing probability while [12] employed WPT in a cooperation-assisted MEC system and obtained the optimal task and power allocation in closed form. Energy transmit beamforming at the AP was studied in [13] to improve the computing performance. Also, in [14], the execution cost was minimized by the Lyapunov optimization.

To reduce the APs' burden and improve the computing experience of UEs, unmanned aerial vehicle (UAV)-assisted MEC has become a promising solution. The UAV-enabled MEC has many advantages, such as easy deployment, flexible locations, line-of-sight (LoS) connections, and moderate computing and caching capabilities, etc. [15], and is a powerful tool to enhance the flexibility and resilience of MEC in handling computation-intensive latency-critical tasks [16]. Joint offloading optimization and trajectory design is a typical problem for the UAV-enabled MEC architecture and has been studied in [17-21]. A UAV-mounted mobile cloud computing was studied in [18], where the UAV helped the UEs compute their offloaded tasks and the total mobile energy consumption was minimized by applying the successive convex approximation (SCA) methods. Later in [19], a UAV-aided offloading scenario was considered at the edges of multiple cells, in which the sum rate of edge users was maximized by optimizing the UAV's 
trajectory and user scheduling. In [20], a two-stage alternative algorithm was applied to solve the problem of maximizing the computation efficiency in a UAV-enabled MEC system. A UAVassisted MEC architecture was investigated in [21], where the UAV acted as a task information relay and an MEC server, and joint optimization on task and bandwidth allocation and UAV's trajectory was investigated to enhance the system performance.

In [22] and [23], the UAV broadcast radio frequency (RF) energy to the UEs and helped them compute offloaded tasks, for maximizing the computation rate of UEs and minimizing the transmitted energy of the UAV, respectively. However, RF energy harvesting may be severely degraded by path loss in practical scenarios. To take advantage of the dominated LoS air-ground links provided by UAVs, a more energy-efficient laser-beamed WPT technology has been utilized in recent wireless-powered UAV-enabled architectures [24,25]. Through narrower energy laser beams, hundred of watts can be harvested at the laser power receiver [26], and the feasibility for laser-charged UAV has been verified by field tests in [27].

\section{B. Our Contributions}

In the UAV-enabled MEC architecture, UEs can relay their data to the UAV to handle their offloaded tasks. Cooperation between the APs and UAVs is sometimes necessary for completing the UEs' tasks when the AP cannot provide reliable connections to some edge users and the size-constrained UAV is resource-limited. Cooperation is even more important in task offloading operated in the millimeter wave (mmWave) bands as large-scale antenna arrays are unlikely to be available due to cost consideration. The blockage issue of mmWave communications can be easily addressed with the assistance of UAVs, for their flexibility and LoS air-ground channel characteristics. To ensure sustainable LoS air-ground UAV links, WPT can be leveraged. In this context, this paper is motivated to propose a wireless-powered UAV-assisted MEC architecture that synergizes the use of AP and UAV via cooperation. In particular, the UAV harvests energy from the AP through laser charging and the UEs harvest energy from the UAV through RF

charging. This architecture is especially beneficial to the cases operating in mmWave bands. To our best knowledge, this is the first MEC work considering the wireless-powered cooperation between AP and UAV, where both laser and RF WPT are utilized.

Our main contributions are summarized as follows:

- Wireless-Powered and UAV-Assisted MEC System with Cooperation between UAV and 
AP: A wireless-powered and UAV-assisted MEC system is considered, where the UAV cooperates with the AP to compute UEs' task-input data with sustainable energy supply. The WPT technology of laser charging is used to provide sustainable energy supply from the AP to UAV, while the WPT technology of RF charging is leveraged for UEs to further harvest energy from the UAV. Here, the UAV plays a key role for serving as an energy relay to provide energy supply for UEs, a moving MEC server to help the UEs compute their tasks, and an information relay to offload the UEs's tasks to the AP.

- Weighted Sum Completed Task-input Bits (WSCTB) Maximization Problem under the Information Causality, Energy Causality, Task and Time Allocation, and UAV's Trajectory Constraints: We provide a WSCTB maximization formulation by jointly considering the optimization of the computation task allocation, time allocation, UAV's energy transmit power and its trajectory, subject to two information causality constraints, two energy causality constraints, the task and time allocation constraints and the UAV's trajectory constraints.

- Three-step Block Coordinate Descending Algorithm: A three-step block coordinate descending algorithm is devised to tackle the non-convex WSCTB maximization problem, by solving three sub-problems iteratively to optimize the task and UAV's energy transmit power allocation, the time allocation, and the UAV's trajectory. In the first sub-problem, the task and UAV's energy transmit power allocation are obtained by leveraging the Lagrange method. In addition, the sub-problem of time allocation is convex and can be solved by standard tools such as CVX [28], while the sub-problem of UAV's trajectory design is also solved by CVX with the aid of the successive convex approximation (SCA) method.

- UAV's Trajectory and Great Performance Improvement: Simulation results are presented to evaluate the performance of the proposed algorithm and illustrate the optimized trajectories of the UAV in different scenarios. The results confirm that the UAV's trajectory and performance highly depend on the AP's location and the UE weights. Besides, the proposed algorithm can achieve great performance gain compared to conventional schemes.

The rest of this paper is organized as follows. Section II first introduces the system model and presents the WSCTB maximization problem. The three-step block coordinate descending algorithm is proposed in Section III. Section IV provides the simulation results to evaluate the proposed algorithm under different scenarios, and we conclude the paper in Section V. 


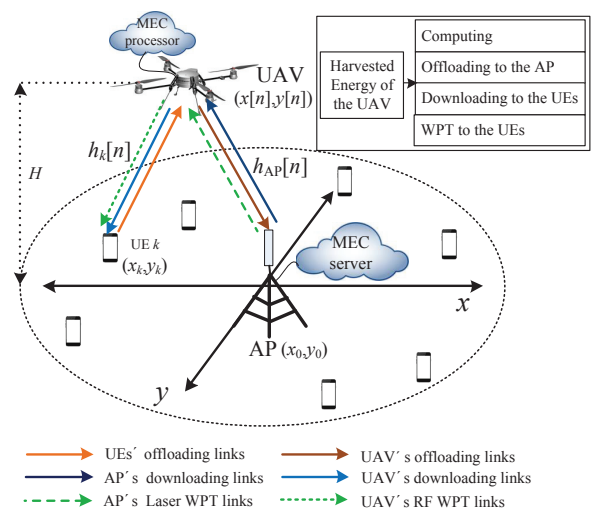

Fig. 1. The wireless-powered MEC architecture with a cooperative UAV, where the UAV harvests laser energy wirelessly from the AP. Besides, the UAV acts as an energy transmitter to offer sustainable RF energy for UEs, and serves as an MEC server and a relay to help the resource-limited UEs compute their offloaded computation tasks or further forward their offloaded tasks to the more powerful processing server at the AP for computing.

\section{System Model AND PROBlem Formulation}

We consider a wireless-powered MEC system with a cooperative UAV, which consists of an AP, a cellular-connected UAV, and $K$ ground UEs, as shown in Fig. 1, where all the nodes have single antenna. It is assumed that the UAV and UEs are equipped with wireless energy-harvesting circuits, communication circuits and computing processors with limited computing capability. In contrast, the grid-powered AP is equipped with an ultra-high performance processing server, capable of high-speed transmission and computation. The AP is also endowed with a laser transmitter for producing high-power laser-like energy beams to the UAV to provide sustainable energy supply for the UAV. Part of the UAV's harvested energy may be broadcast as RF energy to the UEs, while the remaining part is utilized for computing and radio transmissions.

In this paper, each UE is assumed to have a large amount of bit-wise-independent computation task-input data (e.g., for the augmented reality (AR) applications [3]), and the UAV acts as an MEC server as well as a relay to help the UEs compute their task-input data and sometimes further offload their data to the more powerful server at the AP for computing.

We assume that the energy transmissions and the computation task-related information transmissions are operated simultaneously over orthogonal frequency bands. In contrast, different kinds of task-related information transmissions, including the task-input data offloading from UEs to the UAV, from the UAV to the AP, and the task-output data downloading from the UAV 
to UEs, are operated over the same frequency band, with the bandwidth $B$. We further assume that task-related transmissions take place in the mmWave bands. Due to severe blockage for mmWave transmissions, however, the direct links between the UEs and AP are broken, and the UEs cannot harvest energy from the AP or offload their task-input bits to the AP without UAV.

\section{A. Node Locations and Channel Model}

We consider a scenario in which the UAV flies at a constant altitude, denoted as $H$ meters (m), during the task completion time. ${ }^{1}$ The locations of the AP and all the UEs are fixed on the ground with zero altitude. Hence, we can adopt a two-dimensional (2D) Euclidean coordinate system to model the horizontal locations of the UEs, the AP and the UAV, whose coordinates are also measured in meters. Let $\mathcal{K}=\{1, \ldots, K\}$ denote the set of the UEs, and the corresponding horizontal location of UE $k \in \mathcal{K}$ is written as $\mathbf{s}_{k}=\left(x_{k}, y_{k}\right)$. In addition, we use $\mathbf{s}_{0}=\left(x_{0}, y_{0}\right)$ to represent the AP's location. In our considered scenario, the UAV's initial and final horizontal locations are assumed to be preset as $\mathbf{u}_{\mathrm{I}}=\left(x_{\mathrm{I}}, y_{\mathrm{I}}\right)$ and $\mathbf{u}_{\mathrm{F}}=\left(x_{\mathrm{F}}, y_{\mathrm{F}}\right)$, respectively. The total task completion time $T$ seconds (s) is composed of $N$ equal time slots each with a duration of $\tau=T / N$, where $\tau$ is chosen to be sufficiently small such that the UAV's location can be assumed approximately unchanged during each slot. Let $\mathcal{N}=\{1, \ldots, N\}$ denote the set of the $N$ time slots, and the UAV's horizontal location can be further denoted as $\mathbf{u}[n]=\mathbf{u}(n \tau)=(x[n], y[n])$ at the $n$-th time slot with $\mathbf{u}[0]=\mathbf{u}_{\mathrm{I}}$ and $\mathbf{u}[N]=\mathbf{u}_{\mathrm{F}}$. The maximum speed of the UAV is preset as $V_{\max }(\mathrm{m} / \mathrm{s})$ with $V_{\max } \geq\left\|\mathbf{u}_{\mathrm{F}}-\mathbf{u}_{\mathrm{I}}\right\| / T$ to guarantee that at least one feasible trajectory of the UAV exists. The corresponding maximum speed constraint is expressed as ${ }^{2}$

$$
\|\mathbf{v}[n]\|=\frac{1}{\tau}\|\mathbf{u}[n]-\mathbf{u}[n-1]\| \leq V_{\max }, \forall n,
$$

where the UAV is assumed to fly at a constant velocity $\mathbf{v}[n]=(\mathbf{u}[n]-\mathbf{u}[n-1]) / \tau$ during each time slot $n \in \mathcal{N}$.

The wireless channels between the UAV and ground nodes are assumed to be dominated by LoS links $[29]^{3}$, and channel reciprocity holds. Given $h_{0}$ as the channel power gain at a reference distance of $d_{0}=1 \mathrm{~m}$, the channel power gains for the channels between the UAV and the AP as well as UE $k \in \mathcal{K}$ at time slot $n$ can be respectively expressed as

$$
h_{\mathrm{AP}}[n]=\frac{h_{0}}{d_{\mathrm{AP}}^{2}[n]}=\frac{h_{0}}{\left\|\mathbf{u}[n]-\mathbf{s}_{0}\right\|^{2}+H^{2}}, \forall n,
$$

\footnotetext{
${ }^{1} H$ is chosen as the minimum altitude that is appropriate to the work terrain and can avoid buildings without frequent descending and ascending during the task completion time [18-22].

${ }^{2}$ Without loss of generality, the $\forall n$ and $\forall k$ correspond to $\forall n \in \mathcal{N}$ and $\forall k \in \mathcal{K}$ in this paper.

${ }^{3}$ The recent field experiments operated by Qualcomm have verified that the air-ground channels of UAV are dominated by LoS links if proper altitudes are chosen [30], which is applicable in our considered scenario within a short frame $T$.
} 


$$
h_{k}[n]=\frac{h_{0}}{d_{k}^{2}[n]}=\frac{h_{0}}{\left\|\mathbf{u}[n]-\mathbf{s}_{k}\right\|^{2}+H^{2}}, \forall k, n,
$$

where $d_{\mathrm{AP}}[n]$ and $d_{k}[n]$ are the corresponding distances.

\section{B. Computing Methods for UEs' Task-Input Data}

The computation tasks at the UEs involve a large amount of task-input data (e.g., program codes and input parameters), measured by bits. We use the notation $C_{k}$ to represent the amount of required computing resource for computing 1-bit of UE $k$ 's input data (i.e., the number of CPU cycles required). Also, $O_{k} \in(0,1)$ represents the ratio of UE $k$ 's task-output data size to that of the task-input data, which means that computing $I_{k}$ bits of task-input data will generate $O_{k} I_{k}$ bits of task-output data for UE $k \in \mathcal{K}$. Note that the UEs' task-input data are bit-wise independent and can be arbitrarily divided to facilitate parallel trade-offs between local computing at the UEs and computation offloading to the UAV or further to the AP with the help of the UAV.

In summary, the UEs can compute their task-input data in a partial offloading fashion with the following three ways: data computed locally at the UEs, data computed remotely at the UAV by offloading, and data computed remotely at the AP by further offloading. To avoid interference among the UEs during the input data offloading or output data downloading processes by using the latter two offloading methods, we adopt the time-division multiple access (TDMA) protocol. Each slot $n \in \mathcal{N}$ is further divided into $K$ durations, and the operations related to UE $k$ are all executed in the $k$-th duration $t_{k}[n] \in[0, \tau]$ for $k \in \mathcal{K}$, satisfying the following constraint

$$
\sum_{k=1}^{K} t_{k}[n] \leq \tau, \forall n .
$$

1) Data Computed Locally at the UEs: The dynamic voltage and frequency scaling (DVFS) technique is adopted at all the UEs for computing [31]. Denoting the CPU frequency of UE $k$ during slot $n$ as $f_{k}[n]$ (cycles/s), its corresponding computation bits $L_{k}^{\operatorname{loc}}[n]$ and the energy consumption $E_{k}^{\text {loc }}[n]$ during time slot $n$ can be respectively described as

$$
\begin{aligned}
L_{k}^{\mathrm{loc}}[n] & =\tau f_{k}[n] / C_{k}, \forall k, n, \\
E_{k}^{\mathrm{loc}}[n] & =\tau \kappa_{k} f_{k}^{3}[n] \equiv \frac{\kappa_{k} C_{k}^{3}}{\tau^{2}}\left(L_{k}^{\mathrm{loc}}[n]\right)^{3}, \forall k, n,
\end{aligned}
$$

where $\kappa_{k}$ is the effective capacitance coefficient of UE $k$. From the above expressions, we can observe that optimizing $f_{k}[n]$ for DVFS can be equivalently recast into optimizing $L_{k}^{\text {loc }}[n]$. 
2) Data Computed Remotely at the UAV by Offloading: The UEs can resort to the UAV for help by offloading their computation task-input data to the UAV, and part of the offloaded data will be computed at the UAV. Let $P_{k}^{\text {off }}[n]$ and $t_{k}^{\text {off }}[n] \in\left[0, t_{k}[n]\right]$ denote the transmit power for UE $k$ and the allocated time for offloading its task-input data to the UAV at time slot $n$. Then the offloaded task-input bits and the energy consumption of UE $k$ can be calculated as

$$
\begin{aligned}
L_{k}^{\text {off }}[n] & =t_{k}^{\text {off }}[n] B \log _{2}\left(1+\frac{P_{k}^{\text {off }}[n] h_{k}[n]}{N_{0}}\right), \forall k, n, \\
E_{k}^{\text {off }}[n] & =P_{k}^{\text {off }}[n] t_{k}^{\text {off }}[n] \\
& \equiv \frac{N_{0}}{h_{k}[n]}\left(2^{\frac{L_{k}^{\text {off }}[n] / t_{k}^{\text {off }}[n]}{B}}-1\right) t_{k}^{\text {off }}[n], \forall k, n
\end{aligned}
$$

where $N_{0}$ denotes the noise power.

Note that the UAV can help compute UE $k$ 's task for the whole duration allocated to UE $k$, i.e., $t_{k}[n]$, for $n \in \mathcal{N}$, by using its own CPU resources. Assume that the UAV also adopts the DVFS technique to improve its energy efficiency for computing, and the corresponding adjustable CPU frequency is denoted as $f_{\mathrm{U}, k}[n]$. Hence, the completed computation bits and the related energy consumption of UAV for helping UE $k$ during the time slot $n$ can be respectively expressed as

$$
\begin{aligned}
L_{\mathrm{U}, k}[n] & =t_{k}[n] f_{\mathrm{U}, k}[n] / C_{k}, \forall k, n, \\
E_{\mathrm{U}, k}[n] & =t_{k}[n] \kappa_{\mathrm{U}} f_{\mathrm{U}, k}^{3}[n] \\
& \equiv \kappa_{\mathrm{U}} C_{k}^{3} t_{k}[n]\left(\frac{L_{\mathrm{U}, k}[n]}{t_{k}[n]}\right)^{3}, \forall k, n,
\end{aligned}
$$

where $\kappa_{\mathrm{U}}$ is the effective capacitance coefficient of the UAV. It should be noted that computing $L_{\mathrm{U}, k}[n]$ bits of UE $k$ 's task-input data will induce $O_{k} L_{\mathrm{U}, k}[n]$ bits of task-output data, which should be downloaded from the UAV to UE $k$ later.

3) Data Computed Remotely at the AP by Further Offloading: Due to limited resources, the UAV may further offload part of the UEs' offloaded task-input data to the more powerful AP. Let $P_{\mathrm{U}, k}^{\text {off }}[n]$ and $t_{\mathrm{U}, k}^{\text {off }}[n] \in\left[0, t_{k}[n]\right]$ denote the UAV's transmit power and the allocated time for offloading UE $k$ 's task-input data from the UAV to the AP at time slot $n$. Thus, the corresponding further offloaded task-input bits and energy consumption of the UAV at time slot $n$ are given as

$$
\begin{aligned}
L_{\mathrm{U}, k}^{\mathrm{off}}[n] & =t_{\mathrm{U}, k}^{\mathrm{off}}[n] B \log _{2}\left(1+\frac{P_{\mathrm{U}, k}^{\mathrm{off}}[n] h_{\mathrm{AP}}[n]}{N_{0}}\right), \forall k, n, \\
E_{\mathrm{U}, k}^{\mathrm{off}}[n] & =P_{\mathrm{U}, k}^{\mathrm{off}}[n] t_{\mathrm{U}, k}^{\mathrm{off}}[n] \\
& \equiv \frac{N_{0}}{h_{\mathrm{AP}}[n]}\left(2^{\frac{L_{\mathrm{U}, k}^{\mathrm{off}}[n] / t_{\mathrm{U}, k}^{\text {off }}[n]}{{ }^{2}}}-1\right) t_{\mathrm{U}, k}^{\mathrm{off}}[n], \forall k, n .
\end{aligned}
$$

After computing $L_{\mathrm{U}, k}^{\mathrm{off}}[n]$ bits of the UE $k$ 's task-input data at the AP, $O_{k} L_{\mathrm{U}, k}^{\text {off }}[n]$ bits of task-output data will be generated for UE $k$. We assume that the computing time at the AP is negligible. After that, the AP will send UE $k$ 's task-output data back to the UAV also with 


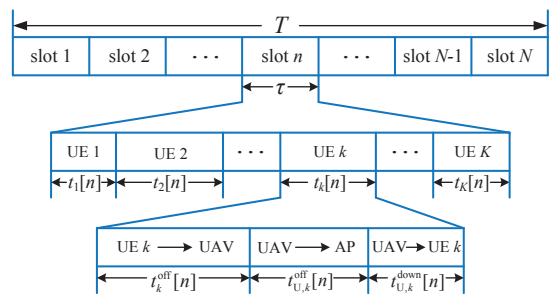

Fig. 2. The TDMA structure of the whole task completion time $T$, which is equally divided into $N$ time slots each with the duration of $\tau=T / N$. In each given slot $n \in \mathcal{N}$, the time allocated to UE $k \in \mathcal{K}$ is $t_{k}[n]$, including UE $k$ 's offloading process to the $\mathrm{UAV}\left(t_{k}^{\mathrm{off}}[n]\right)$, the UAV's offloading process to the AP $\left(t_{\mathrm{U}, k}^{\mathrm{off}}[n]\right)$, and the UAV's downloading process to UE $k\left(t_{\mathrm{U}, k}^{\text {down }}[n]\right)$.

negligible time. In other words, the UAV can receive the task-output data from the AP in the same duration of its offloading process, i.e., $t_{\mathrm{U}, k}^{\mathrm{off}}[n]$.

The generated task-output data at the UAV by applying the latter two offloading methods will then be downloaded back to the corresponding UEs. Let $P_{\mathrm{U}, k}^{\mathrm{down}}[n]$ and $t_{\mathrm{U}, k}^{\mathrm{down}}[n] \in\left[0, t_{k}[n]\right]$ denote the UAV's transmit power and the allocated time for downloading the task-output data from the UAV to UE $k$ at time slot $n$. Hence, the corresponding downloaded task-output bits and the energy consumption of the UAV are found as

$$
\begin{aligned}
L_{\mathrm{U}, k}^{\text {down }}[n] & =t_{\mathrm{U}, k}^{\text {down }}[n] B \log _{2}\left(1+\frac{P_{\mathrm{U}, k}^{\text {down }}[n] h_{k}[n]}{N_{0}}\right), \forall k, n, \\
E_{\mathrm{U}, k}^{\text {down }}[n] & =P_{\mathrm{U}, k}^{\text {down }}[n] t_{\mathrm{U}, k}^{\text {down }}[n] \\
& \equiv \frac{N_{0}}{h_{k}[n]}\left(2^{\frac{L_{\mathrm{U}, k}^{\text {down }}[n] / t_{\mathrm{U}, k}[n]}{\text { down }}}-1\right) t_{\mathrm{U}, k}^{\text {down }}[n], \forall k, n .
\end{aligned}
$$

According to the previous analysis, we have the following time allocation constraint for UE $k$ at time slot $n$

$$
t_{k}^{\text {off }}[n]+t_{\mathrm{U}, k}^{\text {off }}[n]+t_{\mathrm{U}, k}^{\text {down }}[n] \leq t_{k}[n], \forall k, n,
$$

which guarantees that no interference exists among UEs during the task-related information transmissions by leveraging the aforementioned TDMA protocol as shown in Fig. 2.

Assuming that the UAV requires one time slot to process the received data, including the taskinput data from the UEs and the task-output data from the AP. Hence, two information-causality constraints related to the UEs' task-input data and the task-output data should be satisfied. Note that the UAV can only compute or offload UEs' task-input data that has already been received in the previous time slots, which leads to the first information-causality constraint

$$
\sum_{i=2}^{n}\left(L_{\mathrm{U}, k}[i]+L_{\mathrm{U}, k}^{\mathrm{off}}[i]\right) \leq \sum_{i=1}^{n-1} L_{k}^{\mathrm{off}}[i],
$$

for $n \in \mathcal{N}_{2}=\{2, \ldots, N-1\}$ and $k \in \mathcal{K}$. In a similar way, the UAV can only download the task-output data that has already been generated in the previous time slots, either at the UAV or downloaded from the AP, which gives the second information-causality constraint 


$$
\sum_{i=3}^{n} L_{\mathrm{U}, k}^{\mathrm{down}}[i] \leq O_{k} \sum_{i=2}^{n-1}\left(L_{\mathrm{U}, k}[i]+L_{\mathrm{U}, k}^{\mathrm{off}}[i]\right),
$$

for $n \in \mathcal{N}_{3}=\{3, \ldots, N\}$ and $k \in \mathcal{K}$. Note that the UEs should not offload the task-input data at the last two slots, while the UAV should not compute or further offload the input data at the first and the last slots, nor download the output data to the UEs in the first two slots.

\section{Energy Harvesting Model and Energy Constraints}

The energy consumed by the UEs is harvested from the RF charging WPT of the UAV, while the energy consumed by the UAV is fueled from the laser charging WPT of the AP. Assume that a laser transmitter is installed at the AP for transferring laser energy to the UAV with a constant power, denoted as $P_{0}$, and thus the UAV's harvested energy at time slot $n$ through a linear energy harvesting model is given as

$$
\widehat{E}_{\mathrm{U}}[n]=\eta_{\mathrm{U}} g_{\mathrm{AP}}[n] P_{0} \tau, \forall n,
$$

where $\eta_{\mathrm{U}} \in(0,1]$ is the energy conversion efficiency for the UAV. Here, $g_{\mathrm{AP}}[n]=\frac{A \vartheta e^{-\alpha d_{\mathrm{AP}}[n]}}{\left(D+\beta d_{\mathrm{AP}}[n]\right)^{2}}$ is the equivalent channel for laser charing WPT from the AP to the $\mathrm{UAV}^{4}$, where $A$ denotes the area of the laser receiver's telescope or collection lens, $\vartheta$ is the combined transmission receiver optical efficiency, $\alpha$ is the attenuation coefficient of the channel medium in $m^{-1}, D$ indicates the size of the initial laser beam, and $\beta$ is the angular spread $[25,26]$. Suppose that the RF energy transmit power of the UAV at time slot $n$ is $P_{\mathrm{U}}[n]$. Thus the energy consumption of the UAV for WPT and the corresponding harvested energy at UE $k \in \mathcal{K}$ can be expressed as ${ }^{5}$

$$
\begin{aligned}
E_{\mathrm{U}}^{\mathrm{WPT}}[n] & =P_{\mathrm{U}}[n] \tau, \forall n, \\
\widehat{E}_{k}[n] & =\eta_{k} h_{k}[n] P_{\mathrm{U}}[n] \tau, \quad \forall k, n,
\end{aligned}
$$

where $\eta_{k} \in(0,1]$ is the energy conversion efficiency of UE $k$.

The energy consumption of the UEs and the UAV for task computation, offloading and downloading has been obtained in Section II-B. Apart from this, the propulsion energy consumption of the UAV should also be taken into consideration. Supposing that the time slot duration $\tau$ is sufficiently small, the UAV's flying during each slot can be regarded as a straight-and-level flight with constant speed $\mathbf{v}[n]$. Hence, taking a fixed-wing UAV as an example [29,32], its propulsion energy consumption at time slot $n$ can be expressed as

$$
E_{\mathrm{U}}^{\mathrm{prop}}[n]=\tau\left(\zeta_{1}\|\mathbf{v}[n]\|^{3}+\frac{\zeta_{2}}{\|\mathbf{v}[n]\|}\right), \forall n
$$

\footnotetext{
${ }^{4}$ Here $g_{\mathrm{AP}}[n]=h_{\mathrm{AP}}[n]$ if the RF charging is adopted for WPT from the AP to the UAV.

${ }^{5}$ We assume that the input RF power of UEs are within the linear regime of the rectifier, so the linear energy harvesting model is adopted as in [22].
} 
where $\zeta_{1}$ and $\zeta_{2}$ are some fixed parameters related to the UAV specifications.

As a result, we can obtain the total energy consumption of UE $k \in \mathcal{K}$ and the UAV in each time slot $n \in \mathcal{N}$ as

$$
\begin{aligned}
& E_{k}[n]=E_{k}^{\mathrm{loc}}[n]+E_{k}^{\mathrm{off}}[n], \forall k, n, \\
& E_{\mathrm{U}}[n]=\sum_{k=1}^{K}\left(E_{\mathrm{U}, k}[n]+E_{\mathrm{U}, k}^{\mathrm{off}}[n]+E_{\mathrm{U}, k}^{\text {down }}[n]\right)+E_{\mathrm{U}}^{\mathrm{WPT}}[n]+E_{\mathrm{U}}^{\mathrm{prop}}[n], \forall n .
\end{aligned}
$$

Hence, we can respectively obtain the following energy harvesting causality constraints for UE $k$ and the UAV as

$$
\begin{aligned}
& \sum_{i=1}^{n} E_{k}[i] \leq \sum_{i=1}^{n} \widehat{E}_{k}[i], \forall k, n, \\
& \sum_{i=1}^{n} E_{\mathrm{U}}[i] \leq \sum_{i=1}^{n} \widehat{E}_{\mathrm{U}}[i], \forall n .
\end{aligned}
$$

\section{Problem Formulation}

We aim at improving the computing performance by maximizing the WSCTB of UEs, which is equivalent to maximizing the corresponding energy efficiency of the AP. In our model, the UAV's energy transmit power $\left\{P_{\mathrm{U}}[n]\right\}$ at each time slot, the UEs' local computing task sizes $\left\{L_{k}^{\text {loc }}[n]\right\}$, offloading task sizes $\left\{L_{k}^{\text {off }}[n]\right\}$ and the corresponding allocated time $\left\{t_{k}^{\text {off }}[n]\right\}$ at each time slot, the UAV's computing task sizes $\left\{L_{\mathrm{U}, k}[n]\right\}$, further offloading task sizes $\left\{L_{\mathrm{U}, k}^{\mathrm{off}}[n]\right\}$, downloading task sizes $\left\{L_{\mathrm{U}, k}^{\text {down }}[n]\right\}$, and the corresponding allocated time $\left\{t_{k}[n]\right\},\left\{t_{\mathrm{U}, k}^{\text {off }}[n]\right\}$, $\left\{t_{\mathrm{U}, k}^{\text {down }}[n]\right\}$ at each time slot, along with the UAV's trajectory $\{\mathbf{u}[n]\}$ will be optimized jointly to maximize the WSCTB. The problem is formulated as

$$
\begin{aligned}
\max _{\mathbf{L}, \mathbf{t}, \mathbf{P}_{\mathrm{U}}, \mathbf{u}} & \sum_{k=1}^{K} w_{k}\left(\sum_{n=1}^{N} L_{k}^{\text {loc }}[n]+\sum_{n=1}^{N-2} L_{k}^{\text {off }}[n]\right) \\
\text { s.t. } \quad & \sum_{k=1}^{K} t_{k}[n] \leq \tau, \forall n, t_{k}[n] \geq 0, \forall n, k, \\
& t_{k}^{\text {off }}[n]+t_{\mathrm{U}, k}^{\text {off }}[n]+t_{\mathrm{U}, k}^{\text {down }}[n] \leq t_{k}[n], \forall n, k, \\
& \sum_{i=2}^{n}\left(L_{\mathrm{U}, k}[i]+L_{\mathrm{U}, k}^{\text {off }}[i]\right) \leq \sum_{i=1}^{n-1} L_{k}^{\text {off }}[i], \forall n \in \mathcal{N}_{2}, \forall k, \\
& \sum_{i=3}^{n} L_{\mathrm{U}, k}^{\text {down }}[i] \leq O_{k} \sum_{i=2}^{n-1}\left(L_{\mathrm{U}, k}[i]+L_{\mathrm{U}, k}^{\text {off }}[i]\right), \forall n \in \mathcal{N}_{3}, \forall k, \\
& \sum_{n=2}^{N-1}\left(L_{\mathrm{U}, k}[n]+L_{\mathrm{U}, k}^{\text {off }}[n]\right)=\sum_{n=1}^{N-2} L_{k}^{\text {off }}[n], \forall k, \\
& \sum_{n=3}^{N} L_{\mathrm{U}, k}^{\text {down }}[n]=O_{k} \sum_{n=2}^{N-1}\left(L_{\mathrm{U}, k}[n]+L_{\mathrm{U}, k}^{\text {off }}[n]\right), \forall k, \\
& \sum_{i=1}^{n} E_{k}[i](\mathbf{L}, \mathbf{t}, \mathbf{u}) \leq \eta_{k} \tau \sum_{i=1}^{n} h_{k}[i] P_{\mathrm{U}}[i], \forall n, k,
\end{aligned}
$$




$$
\begin{aligned}
& \sum_{i=1}^{n} E_{\mathrm{U}}[i]\left(\mathbf{L}, \mathbf{t}, \mathbf{P}_{\mathrm{U}}, \mathbf{u}\right) \leq \eta_{\mathrm{U}} \tau P_{0} \sum_{i=1}^{n} g_{\mathrm{AP}}[i], \forall n, \\
& P_{\mathrm{U}}[n] \geq 0, \forall n, \\
& L_{k}^{\text {loc }}[n] \geq 0, \forall n, k, \\
& L_{k}^{\text {off }}[N-1]=L_{k}^{\text {off }}[N]=0, L_{k}^{\text {off }}[n] \geq 0, \forall n \in \mathcal{N}_{1}, \forall k, \\
& L_{\mathrm{U}, k}[1]=L_{\mathrm{U}, k}[N]=0, L_{\mathrm{U}, k}[n] \geq 0, \forall n \in \mathcal{N}_{2}, \forall k, \\
& L_{\mathrm{U}, k}^{\text {off }}[1]=L_{\mathrm{U}, k}^{\text {off }}[N]=0, \quad L_{\mathrm{U}, k}^{\text {off }}[n] \geq 0, \forall n \in \mathcal{N}_{2}, \forall k, \\
& L_{\mathrm{U}, k}^{\text {down }}[1]=L_{\mathrm{U}, k}^{\text {down }}[2]=0, L_{\mathrm{U}, k}^{\text {down }}[n] \geq 0, \forall n \in \mathcal{N}_{3}, \forall k, \\
& t_{k}^{\text {off }}[N-1]=t_{k}^{\text {off }}[N]=0, t_{k}^{\text {off }}[n] \geq 0, \forall n \in \mathcal{N}_{1}, \forall k, \\
& t_{\mathrm{U}, k}^{\text {off }}[1]=t_{\mathrm{U}, k}^{\text {off }}[N]=0, t_{\mathrm{U}, k}^{\text {off }}[n] \geq 0, \forall n \in \mathcal{N}_{2}, \forall k, \\
& t_{\mathrm{U}, k}^{\text {down }}[1]=t_{\mathrm{U}, k}^{\text {down }}[2]=0, t_{\mathrm{U}, k}^{\text {down }}[n] \geq 0, \forall n \in \mathcal{N}_{3}, \forall k, \\
& \mathbf{u}_{[0]}[0]=\mathbf{u}_{\mathrm{I}}, \quad \mathbf{u}[N]=\mathbf{u}_{\mathrm{F}}, \\
& \|\mathbf{u}[n]-\mathbf{u}[n-1]\| \leq V_{\max } \tau, \forall n,
\end{aligned}
$$

where $\left\{w_{k} \geq 0\right\}_{k \in \mathcal{K}}$ denotes the set of UEs' weights for their completed task-input bits and $\mathcal{N}_{1}=\{1, \ldots, N-2\}$. The optimization variables include $\mathbf{L} \triangleq\left\{L_{k}^{\text {loc }}[n], L_{k}^{\text {off }}[n], L_{\mathrm{U}, k}[n], L_{\mathrm{U}, k}^{\text {off }}[n]\right.$, $\left.L_{\mathrm{U}, k}^{\text {down }}[n]\right\}_{k \in \mathcal{K}, n \in \mathcal{N}}$ and $\mathbf{t} \triangleq\left\{t_{k}[n], t_{k}^{\text {off }}[n], t_{\mathrm{U}, k}^{\text {off }}[n], t_{\mathrm{U}, k}^{\text {down }}[n]\right\}_{k \in \mathcal{K}, n \in \mathcal{N}}$ respectively denoting the sets of the computation task allocation variables and the time allocation variables, $\mathbf{P}_{\mathrm{U}} \triangleq\left\{P_{\mathrm{U}}[n]\right\}_{n \in \mathcal{N}}$ being the set of the UAV's energy transmit power at each time slot, and $\mathbf{u} \triangleq\{\mathbf{u}[n]\}_{n \in \mathcal{N}}$ indicating the set of UAV's horizontal locations, i.e., the trajectory of UAV.

\section{Algorithm Design}

The problem (26) is non-convex because of the energy causality constraints (26h) and (26i), where the variables $\mathbf{L}, \mathbf{t}, \mathbf{P}_{\mathrm{U}}$ are strongly coupled with the UAV's trajectory $\mathbf{u}$. Besides, the computation task allocation variables $\left\{L_{k}^{\text {off }}[n]\right\},\left\{L_{\mathrm{U}, k}[n]\right\},\left\{L_{\mathrm{U}, k}^{\text {off }}[n]\right\},\left\{L_{\mathrm{U}, k}^{\text {down }}[n]\right\}$ in $\mathbf{L}$ are highly coupled with the corresponding time allocation variables $\left\{t_{k}^{\text {off }}[n]\right\},\left\{t_{k}[n]\right\},\left\{t_{\mathrm{U}, k}^{\text {off }}[n]\right\},\left\{t_{\mathrm{U}, k}^{\text {down }}[n]\right\}$ in $\mathbf{t}$, respectively. To tackle the challenges, we propose a three-step block coordinate descending algorithm to optimize the computational task allocation variables in L and the UAV's energy transmit power in $\mathbf{P}_{\mathrm{U}}$, the time allocation variables in $\mathbf{t}$, and the UAV's trajectory $\mathbf{u}$ iteratively.

In the $(\chi+1)$-th $(\chi=0,1,2, \ldots)$ iteration, we first optimize $\mathbf{L}$ and $\mathbf{P}_{\mathbf{U}}$ with $\mathbf{t}$ and $\mathbf{u}$ fixed as the optimized values achieved in the $\chi$-th iteration, denoted as $\mathbf{t}_{\chi}$ and $\mathbf{u}_{\chi}$, and the obtained 
solutions are written as $\overline{\mathbf{L}}_{\chi+1}$ and $\mathbf{P}_{\mathrm{U}, \chi+1}$, where the task allocation parameters obtained in $\overline{\mathbf{L}}_{\chi+1}$ are temporary given $\mathbf{t}_{\chi}$ and $\mathbf{u}_{\chi}$. Then we optimize the time allocation $\mathbf{t}$ with the given $\mathbf{u}_{\chi}$ and $\overline{\mathbf{L}}_{\chi+1}, \mathbf{P}_{\mathrm{U}, \chi+1}$, to obtain the solution $\mathbf{t}_{\chi+1}$. Finally, the UAV's trajectory $\mathbf{u}$ is optimized with the given $\overline{\mathbf{L}}_{\chi+1}, \mathbf{P}_{\mathrm{U}, \chi+1}$ and $\mathbf{t}_{\chi+1}$, and the obtained solution is denoted as $\mathbf{u}_{\chi+1}$. The final $\mathbf{L}_{\chi+1}$ for the $(\chi+1)$-th iteration can be obtained with $\mathbf{t}_{\chi+1}$ and $\mathbf{u}_{\chi+1}$. The details of the three-step block coordinate descending algorithm for solving the WSCTB maximization problem (26) at the $(\chi+1)$-th iteration with $\mathbf{t}_{\chi}$ and $\mathbf{u}_{\chi}$ are presented in the following subsections.

\section{A. Computation Task and UAV's WPT Power Allocation}

Here, we consider a sub-problem of the original WSCTB maximization problem (26) by fixing the time allocation $\mathbf{t}$ and the UAV's trajectory $\mathbf{u}$ as $\mathbf{t}_{\chi}$ and $\mathbf{u}_{\chi}$, denoted as the computation task and UAV's power allocation (CTUPA) problem. With the given $\mathbf{u}_{\chi}$, the time-dependent channels defined in (2), (3) and (18) are known as $\left\{h_{\mathrm{AP}, \chi}[n]\right\}_{n \in \mathcal{N}},\left\{h_{k, \chi}[n]\right\}_{k \in \mathcal{K}, n \in \mathcal{N}}$ and $\left\{g_{\mathrm{AP}, \chi}[n]\right\}_{n \in \mathcal{N}}$. Hence, the CTUPA problem (P1) can be expressed as

$$
\begin{aligned}
\text { (P1) : } \max _{\mathbf{L}, \mathbf{P}_{\mathbf{U}}} & \sum_{k=1}^{K} w_{k}\left(\sum_{n=1}^{N} L_{k}^{\mathrm{loc}}[n]+\sum_{n=1}^{N-2} L_{k}^{\mathrm{off}}[n]\right) \\
\text { s.t. } & (26 \mathrm{~d})-(26 \mathrm{o}) .
\end{aligned}
$$

By fixing $\mathbf{t}_{\chi}$ and $\mathbf{u}_{\chi}$, the energy-causality constraints (26h) and (26i) have been turned into convex forms, making (P1) a convex optimization problem with respect to (w.r.t.) $\mathbf{L}$ and $\mathbf{P}_{\mathrm{U}}$. Here, the Lagrange method [33] is leveraged to solve problem (P1).

Theorem 1. The optimal solution of problem $(P 1)$ corresponding to $U E k \in \mathcal{K}$ is given in (28)-(33) (where $P_{\mathrm{U}, \chi+1}^{\mathrm{low}}[n]$ given by (34) and $P_{\mathrm{U}, \chi+1}^{\mathrm{up}}[n]$ given by (35) respectively denote a lower bound and an upper bound solution of the UAV's energy transmit power in (33)).

$$
\begin{aligned}
L_{k, \chi+1}^{\text {loc }}[n] & =\frac{\tau}{C_{k}} \sqrt{\frac{w_{k}}{3 \kappa_{k} C_{k} \sum_{i=n}^{N} \xi_{k, i}^{*}}}, n \in \mathcal{N}, \\
\bar{L}_{k, \chi+1}^{\text {off }}[n] & =\left\{\begin{array}{l}
B t_{k, \chi}^{\text {off }}[n]\left[\log _{2}\left[B h_{k, \chi}[n]\left(w_{k}+\lambda_{k}^{*}+\sum_{i=n+1}^{N-1} \gamma_{k, i}^{*}\right)\right]^{+}-\log _{2}\left(N_{0} \ln 2 \sum_{i=n}^{N} \xi_{k, i}^{*}\right)\right]^{+}, n \in \mathcal{N}_{1}, \\
0, \quad n=N-1 \text { or } N,
\end{array}\right. \\
\bar{L}_{\mathrm{U}, k, \chi+1}[n] & =\left\{\begin{array}{l}
t_{k, \chi}[n] \sqrt{\frac{1}{3 \kappa_{\mathrm{U}} C_{k}^{3} \sum_{i=n}^{N} \rho_{i}^{*}}\left[O_{k} \sum_{i=n+1}^{N} \mu_{k, i}^{*}-\sum_{i=n}^{N-1} \gamma_{k, i}^{*}-O_{k} \nu_{k}^{*}-\lambda_{k}^{*}\right]^{+}}, n \in \mathcal{N}_{2}, \\
0, \quad n=1 \text { or } N,
\end{array}\right.
\end{aligned}
$$




$$
\begin{aligned}
& \bar{L}_{\mathrm{U}, k, \chi+1}^{\mathrm{off}}[n]=\left\{\begin{aligned}
& B t_{\mathrm{U}, k, \chi}^{\mathrm{off}}[n] {\left[\log _{2}\left[B h_{\mathrm{AP}, \chi}[n]\left(O_{k} \sum_{i=n+1}^{N} \mu_{k, i}^{*}-\sum_{i=n}^{N-1} \gamma_{k, i}^{*}-O_{k} \nu_{k}^{*}-\lambda_{k}^{*}\right)\right]^{+}\right.} \\
&\left.-\log _{2}\left(N_{0} \ln 2 \sum_{i=n}^{N} \rho_{i}^{*}\right)\right]^{+}, n \in \mathcal{N}_{2}, \\
& 0, \quad n=1 \text { or } N,
\end{aligned}\right. \\
& \bar{L}_{\mathrm{U}, k, \chi+1}^{\text {down }}[n]=\left\{\begin{array}{l}
B t_{\mathrm{U}, k, \chi}^{\mathrm{down}}[n]\left[\log _{2}\left[B h_{k, \chi}[n]\left(\nu_{k}^{*}-\sum_{i=n}^{N} \mu_{k, i}^{*}\right)\right]^{+}-\log _{2}\left(N_{0} \ln 2 \sum_{i=n}^{N} \rho_{i}^{*}\right)\right]^{+}, n \in \mathcal{N}_{3}, \\
0, \quad n=1 \text { or } 2,
\end{array}\right. \\
& P_{\mathrm{U}, \chi+1}[n]=\left\{\begin{array}{l}
P_{\mathrm{U}, \chi+1}^{\mathrm{up}}[n], \quad \text { if } \sum_{i=n}^{N} \rho_{i}^{*}<\sum_{k=1}^{K}\left(\eta_{k} h_{k, \chi}[n] \sum_{i=n}^{N} \xi_{k, i}^{*}\right), n \in \mathcal{N}, \\
P_{\mathrm{U}, \chi+1}^{\text {low }}[n], \quad \text { otherwie, } n \in \mathcal{N},
\end{array}\right. \\
& P_{\mathrm{U}, \chi+1}^{\text {low }}[n]=\max \left\{\left[\frac{\sum_{i=1}^{n} E_{k}[i]\left(\overline{\mathbf{L}}_{\chi+1}, \mathbf{t}_{\chi}, \mathbf{u}_{\chi}\right)}{\eta_{k} \tau h_{k, \chi}[n]}-\frac{\sum_{i=1}^{n-1} h_{k, \chi}[i] P_{\mathrm{U}, \chi+1}^{\text {low }}[i]}{h_{k, \chi}[n]}\right]^{+}\right\}_{k \in \mathcal{K}}, \\
& P_{\mathrm{U}, \chi+1}^{\mathrm{up}}[n]=\left[\frac{1}{\tau} \sum_{i=1}^{n}\left(\eta_{\mathrm{U}} \tau P_{0} g_{\mathrm{AP}, \chi}[i]-E_{\mathrm{U}}^{\mathrm{self}}[i]\left(\overline{\mathbf{L}}_{\chi+1}, \mathbf{t}_{\chi}, \mathbf{u}_{\chi}\right)\right)-\sum_{i=1}^{n-1} P_{\mathrm{U}, \chi+1}^{\mathrm{up}}[i]\right]^{+} \\
& E_{\mathrm{U}}^{\text {self }}[n]\left(\overline{\mathbf{L}}_{\chi+1}, \mathbf{t}_{\chi}, \mathbf{u}_{\chi}\right)=\sum_{k=1}^{K}\left(E_{\mathrm{U}, k}[n]+E_{\mathrm{U}, k}^{\mathrm{off}}[n]+E_{\mathrm{U}, k}^{\text {down }}[n]\right)+E_{\mathrm{U}}^{\text {prop }}[n] .
\end{aligned}
$$

Here, $\gamma_{k, n}^{*} \geq 0, \mu_{k, n}^{*} \geq 0, \xi_{k, n}^{*} \geq 0, \rho_{n}^{*} \geq 0$ for $k \in \mathcal{K}, n \in \mathcal{N}$ respectively indicate the optimal Lagrange multipliers associated with the inequality constraints (26d), (26e), (26h), (26i) in problem $(P 1)$, while $\lambda_{k}^{*}$ and $\nu_{k}^{*}$ for $k \in \mathcal{K}$ are respectively the optimal Lagrange multipliers associated with the equality constraints (26f) and (26g) in problem (P1). We then have $\overline{\mathbf{L}}_{\chi+1}=$ $\left\{L_{k, \chi+1}^{\text {loc }}[n], \bar{L}_{k, \chi+1}^{\text {off }}[n], \bar{L}_{\mathrm{U}, k, \chi+1}[n], \bar{L}_{\mathrm{U}, k, \chi+1}^{\text {off }}[n], \bar{L}_{\mathrm{U}, k, \chi+1}^{\text {down }}[n]\right\}$, and $\mathbf{P}_{\mathrm{U}, \chi+1}=\left\{P_{\mathrm{U}, \chi+1}[n]\right\}$.

Proof. See Appendix A.

Remark 1. The expressions of $L_{k, \chi+1}^{\mathrm{loc}}[n]$ and $\bar{L}_{k, \chi+1}^{\mathrm{off}}[n]$ in Theorem 1 indicate that the weight

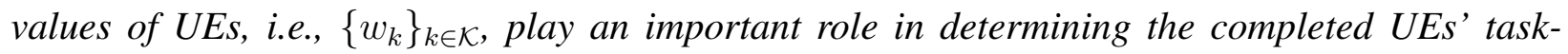
input data. The UEs with larger weight values are more likely to complete more task-input data through both local computing and computation offloading.

Remark 2. The expressions of $\bar{L}_{k, \chi+1}^{\mathrm{off}}[n]$ and $\bar{L}_{\mathrm{U}, k, \chi+1}^{\mathrm{off}}[n]$ in Theorem 1 further show that the UEs with good offloading (or downloading) channels at any time slot, i.e., $h_{k, \chi}[n]$, are more likely to offload more computation bits for remote computing at the UAV or the AP.

Next, we will utilize the subgradient method [34] to obtain the Lagrange multipliers in $\gamma^{*}=$ $\left\{\gamma_{k, n}^{*}\right\}, \boldsymbol{\mu}^{*}=\left\{\mu_{k, n}^{*}\right\}, \boldsymbol{\xi}^{*}=\left\{\xi_{k, n}^{*}\right\}$ and $\boldsymbol{\rho}^{*}=\left\{\rho_{n}^{*}\right\}$, related to the inequality constraints (26d), 
(26e), (26h) and (26i), as described in the following Lemma 1. Furthermore, the optimal Lagrange multipliers in $\boldsymbol{\lambda}^{*}=\left\{\lambda_{k}^{*}\right\}$ and $\boldsymbol{\nu}^{*}=\left\{\nu_{k}^{*}\right\}$ related to the equality constraints (26f) and (26g) can be obtained by leveraging the bi-section search method, as summarized in Lemma 2.

Lemma 1. At the $(j+1)$-th $(j=1,2, \ldots)$ iteration of the subgradient algorithm, the corresponding Lagrange multipliers $\left\{\gamma_{k, n}\right\},\left\{\mu_{k, n}\right\},\left\{\xi_{k, n}\right\},\left\{\rho_{n}\right\}$ are given as ${ }^{6}$

$$
\begin{aligned}
& \gamma_{k, n, j+1}=\left[\gamma_{k, n, j}-\varepsilon_{j}^{(\gamma)} \Delta \gamma_{k, n, j}\right]^{+}, \forall n \in \mathcal{N}_{2}, \forall k, \\
& \mu_{k, n, j+1}=\left[\mu_{k, n, j}-\varepsilon_{j}^{(\mu)} \Delta \mu_{k, n, j}\right]^{+}, \forall n \in \mathcal{N}_{3}, \forall k, \\
& \xi_{k, n, j+1}=\left[\xi_{k, n, j}-\varepsilon_{j}^{(\xi)} \Delta \xi_{k, n, j}\right]^{+}, \forall n \in \mathcal{N}, \forall k, \\
& \rho_{n, j+1}=\left[\rho_{n, j}-\varepsilon_{j}^{(\rho)} \Delta \rho_{n, j}\right]^{+}, \forall n,
\end{aligned}
$$

where $\varepsilon_{j}^{(\lambda)}, \varepsilon_{j}^{(\mu)}, \varepsilon_{j}^{(\xi)}$ and $\varepsilon_{j}^{(\rho)}$ respectively denote the iterative steps for obtaining the Lagrange multipliers in $\gamma, \mu, \xi$ and $\rho$ at the $j$-th iteration, and the subgradients are expressed as

$$
\begin{aligned}
& \Delta \gamma_{k, n, j}=\sum_{i=1}^{n-1} \bar{L}_{k, j}^{\mathrm{off}}[i]-\sum_{i=2}^{n}\left(\bar{L}_{\mathrm{U}, k, j}[i]+\bar{L}_{\mathrm{U}, k, j}^{\mathrm{off}}[i]\right), \\
& \Delta \mu_{k, n, j}=O_{k} \sum_{i=2}^{n-1}\left(\bar{L}_{\mathrm{U}, k, j}[i]+\bar{L}_{\mathrm{U}, k, j}^{\mathrm{off}}[i]\right)-\sum_{i=3}^{n} \bar{L}_{\mathrm{U}, k, j}^{\mathrm{down}}[i], \\
& \Delta \xi_{k, n, j}=\eta_{k} \tau \sum_{i=1}^{n} h_{k, \chi}[i] P_{\mathrm{U}, j}[i]-\sum_{i=1}^{n} E_{k}[i]\left(\overline{\mathbf{L}}_{j}, \mathbf{t}_{\chi}, \mathbf{u}_{\chi}\right), \\
& \Delta \rho_{n, j}=\eta_{\mathrm{U}} \tau P_{0} \sum_{i=1}^{n} g_{\mathrm{AP}, \chi}[i]-\sum_{i=1}^{n} E_{\mathrm{U}}[i]\left(\overline{\mathbf{L}}_{j}, \mathbf{P}_{\mathrm{U}, j}, \mathbf{t}_{\chi}, \mathbf{u}_{\chi}\right) .
\end{aligned}
$$

Note that $\left\{L_{k, j}^{\text {loc }}[n]\right\},\left\{\bar{L}_{k, j}^{\text {off }}[n]\right\},\left\{\bar{L}_{\mathrm{U}, k, j}[n]\right\},\left\{\bar{L}_{\mathrm{U}, k, j}^{\mathrm{off}}[n]\right\},\left\{\bar{L}_{\mathrm{U}, k, j}^{\mathrm{down}}[n]\right\}$, and $\left\{P_{\mathrm{U}, j}[n]\right\}$ are the task allocation and the UAV's energy transmit power obtained from Theorem 1 with the Lagrange multipliers obtained at the $j$-th iteration.

Remark 3. During each iteration of the subgradient method, the UAV's energy transmit power $\left\{P_{\mathrm{U}, j+1}[n]\right\}$ plays an important role in trading off maximizing the WSCTB and satisfying the constraints. If the value of $\sum_{i=n}^{N} \xi_{k, i, j}$ is relatively large, leading to small values of $L_{k, j+1}^{\text {loc }}[n]$ and $\bar{L}_{k, j+1}^{\mathrm{off}}[n], P_{\mathrm{U}, j+1}[n]$ prefers to choose its upper bound according to (33) to maximize the WSCTB of UEs. If the value of $\sum_{i=n}^{N} \rho_{i, j}$ is relatively large, leading to small values of $\bar{L}_{\mathrm{U}, k, j+1}[n]$, $\bar{L}_{\mathrm{U}, k, j+1}^{\mathrm{off}}[n]$ and $\bar{L}_{\mathrm{U}, k, j}^{\mathrm{down}}[n], P_{\mathrm{U}, j+1}[n]$ tends to choose its lower bound according to (33), where more UAV's energy will be used to improve these three values to satisfy the task allocation constraints (26d)-(26g).

\footnotetext{
${ }^{6}$ In Lemma 1, Lemma 2 and the corresponding proofs, we omit the index $\chi+1$ at the subscripts of $\overline{\mathbf{L}}_{\chi+1}$ and $\mathbf{P}_{\mathrm{U}, \chi+1}$ as well as the individual optimized parameters in $\overline{\mathbf{L}}_{\chi+1}$ and $\mathbf{P}_{\mathrm{U}, \chi+1}$ for conciseness.
} 
Lemma 2. Based on the obtained $\boldsymbol{\gamma}_{j+1}, \boldsymbol{\mu}_{j+1}, \boldsymbol{\xi}_{j+1}$ and $\boldsymbol{\rho}_{j+1}$ in Lemma 1, we can then obtain $\boldsymbol{\lambda}_{j+1}$ and $\boldsymbol{\nu}_{j+1}$ by bi-section search of $\left\{\sum_{n=1}^{N-2} \bar{L}_{k, j+1}^{\mathrm{off}}[n] \in\left[\bar{L}_{k, j+1}^{\min }, \bar{L}_{k, j+1}^{\max }\right]\right\}_{k \in \mathcal{K}}$ at the iteration. For a given $\sum_{n=1}^{N-2} \bar{L}_{k, j+1}^{\mathrm{off}}[n]$, the corresponding $\lambda_{k, j+1}$ and $\nu_{k, j+1}$ can be achieved with another two bi-section searches within $\lambda_{k, j+1} \in\left[\lambda_{k, j+1}^{\min }, \lambda_{k, j+1}^{\max }\right]$ and $\nu_{k, j+1} \in\left[\nu_{k, j+1}^{\min }, \nu_{k, j+1}^{\max }\right]$ with

$$
\begin{aligned}
\lambda_{k, j+1}^{\min } & =-w_{k}-\widehat{\gamma}_{k, 1, j+1}, \\
\lambda_{k, j+1}^{\max } & =-\gamma_{k, N-1, j+1}+O_{k} \widehat{\mu}_{k, 2, j+1}, \\
\nu_{k, j+1}^{\min } & =\mu_{k, N, j+1}, \\
\nu_{k, j+1}^{\max } & =\widehat{\mu}_{k, 2, j+1}+\left(w_{k}+\widehat{\gamma}_{k, 1, j+1}\right) / O_{k},
\end{aligned}
$$

where $\widehat{\gamma}_{k, n}=\sum_{i=n+1}^{N-1} \gamma_{k, i}$ and $\widehat{\mu}_{k, n}=\sum_{i=n+1}^{N} \mu_{k, i}$. In fact, we have $\bar{L}_{k, j+1}^{\min }=\min \left\{V_{k, j+1}^{\mathrm{C} 1}\left(\lambda_{k, j+1}^{\min }\right)\right.$, $\left.V_{k, j+1}^{\mathrm{C} 2}\left(\nu_{k, j+1}^{\min }\right), V_{k, j+1}^{\mathrm{C} 3}\left(\lambda_{k, j+1}^{\max }, \nu_{k, j+1}^{\max }\right)\right\}, \bar{L}_{k, j+1}^{\max }=\max \left\{V_{k, j+1}^{\mathrm{C} 1}\left(\lambda_{k, j+1}^{\max }\right), V_{k, j+1}^{\mathrm{C} 2}\left(\nu_{k, j+1}^{\max }\right), V_{k, j+1}^{\mathrm{C} 3}\left(\lambda_{k, j+1}^{\min }\right.\right.$, $\left.\left.\nu_{k, j+1}^{\min }\right)\right\}$, in which the $V_{k, j+1}^{\mathrm{C} 1}, V_{k, j+1}^{\mathrm{C} 2}$ and $V_{k, j+1}^{\mathrm{C} 3}$ are the right side values of (B.1), (B.2) and (B.3) in Appendix B w.r.t. $\lambda_{k, j+1}$ and $\nu_{k, j+1}$.

The finally obtained $\lambda_{k, j+1}$ and $\nu_{k, j+1}$ corresponding to the optimal $\sum_{n=1}^{N-2} \bar{L}_{k, j+1}^{\text {off }}[n]$ should satisfy that $\sum_{n=1}^{N-2} \bar{L}_{k, j+1}^{\text {off }}[n]=V_{k, \mathrm{C} 1}\left(\lambda_{k, j+1}\right)=V_{k, \mathrm{C} 2}\left(\nu_{k, j+1}\right)=V_{k, \mathrm{C} 3}\left(\lambda_{k, j+1}, \nu_{k, j+1}\right)$, which indicates the termination of the bi-section searches.

Proof. See Appendix B.

Through Lemma 1 and Lemma 2, we can finally obtain the optimal dual variables $\boldsymbol{\gamma}^{*}, \boldsymbol{\mu}^{*}, \boldsymbol{\xi}^{*}, \boldsymbol{\rho}^{*}$ and $\lambda^{*}, \nu^{*}$, when the subgradient algorithm converges and the bi-section searches terminate, leading to a guaranteed convergence [33].

Lemma 3. With the computation task allocation parameters $\left\{\bar{L}_{k, \chi+1}^{\text {off }}[n]\right\},\left\{\bar{L}_{\mathrm{U}, k, \chi+1}^{\text {off }}[n]\right\}$, and $\left\{\bar{L}_{\mathrm{U}, k, \chi+1}^{\text {down }}[n]\right\}$ in Theorem 1, we can further obtain the corresponding transmit power according to (8), (12) and (14), which are given by ${ }^{7}$

$$
\begin{aligned}
& P_{k, \chi+1}^{\text {off }}[n]=\frac{N_{0}}{h_{k}[n]}\left(2^{\frac{L_{k, \chi}^{\text {off }}[n] / t_{k, \chi}^{\text {off }}[n]}{B}}-1\right), \\
& P_{\mathrm{U}, k, \chi+1}^{\text {off }}[n]=\frac{N_{0}}{h_{\mathrm{AP}}[n]}\left(2^{\frac{\bar{L}_{\mathrm{U}, k, \chi+1}^{\text {off }}[n] / t_{\mathrm{U}, k, \chi}^{\text {off }}}{B}}-1\right), \\
& P_{\mathrm{U}, k, \chi+1}^{\text {down }}[n]=\frac{N_{0}}{h_{k}[n]}\left(2^{\frac{\bar{L}_{\mathrm{U}, k, \chi+1}^{\text {down }}[n] / t_{\mathrm{U}, k, \chi}^{\text {down }}[n]}{B}}-1\right) .
\end{aligned}
$$

Besides, with $\left\{\bar{L}_{\mathrm{U}, k, \chi+1}[n]\right\}$, we can further obtain the corresponding CPU frequency of the UAV through (9) as

${ }^{7}$ We omit $\forall n \in \mathcal{N}_{1}, \forall k$ in (49), $\forall n \in \mathcal{N}_{2}, \forall k$ in (50), and $\forall n \in \mathcal{N}_{3}, \forall k$ in (51) for the sake of space limitation. Similarly, this rule also applies to the equations (58), (59), (60). 


$$
f_{\mathrm{U}, k, \chi+1}[n]=C_{k} \frac{\bar{L}_{\mathrm{U}, k, \chi+1}[n]}{t_{k, \chi}[n]}, \forall n \in \mathcal{N}_{2}, \forall k
$$

To help our discussion, we split the parameters in $\overline{\mathbf{L}}_{\chi+1}$ into two sets as $\mathbf{L}_{\chi+1}^{\text {loc }}=\left\{L_{k, \chi+1}^{\text {loc }}[n]\right\}$ and $\mathbf{R}_{\chi+1}^{\mathrm{res}}=\left\{P_{k, \chi+1}^{\mathrm{off}}[n], P_{\mathrm{U}, k, \chi+1}^{\mathrm{off}}[n], P_{\mathrm{U}, k, \chi+1}^{\text {down }}[n], f_{\mathrm{U}, k, \chi+1}[n]\right\}$, which are respectively the parameter sets related to task-input data sizes for local computing as well as the radio/computing resources required for remote computation offloading. In the next sub-section, the time allocation will be optimized with the fixed $\mathbf{L}_{\chi+1}^{\text {loc }}, \mathbf{R}_{\chi+1}^{\text {res }}, \mathbf{P}_{\mathrm{U}, \chi+1}$ and $\mathbf{u}_{\chi}$ to obtain $\mathbf{t}_{\chi+1}$.

\section{B. Time Allocation}

In this section, another sub-problem of the original WSCTB maximization problem (26) in the $(\chi+1)$-th iteration is addressed to optimize $\mathbf{t}$, denoted as the time allocation problem, with the UAV's trajectory $\mathbf{u}_{\chi}$, the optimized task allocation related parameters in $\mathbf{L}_{\chi+1}^{\text {loc }}$ and $\mathbf{R}_{\chi+1}^{\text {res }}$, and the UAV's WPT power allocation $\mathbf{P}_{\mathrm{U}, \chi+1}$. In this case, we can re-express UE $k$ 's offloading bits, the UAV's offloading, downloading, and computing bits as functions of $\mathbf{t}$ by substituting $\left\{P_{k, \chi+1}^{\text {off }}[n]\right\},\left\{P_{\mathrm{U}, k, \chi+1}^{\text {off }}[n]\right\},\left\{P_{\mathrm{U}, k, \chi+1}^{\text {down }}[n]\right\}$, and $\left\{f_{\mathrm{U}, k, \chi+1}[n]\right\}$ in expressions (49), (50), (51), and (52) into the expressions (7), (11), (13), and (9) respectively, which are given as

$$
\begin{aligned}
& L_{k}^{\text {off }}[n]\left(t_{k}^{\text {off }}[n]\right)=t_{k}^{\text {off }}[n] \frac{\bar{L}_{k, \chi+1}^{\text {off }}[n]}{t_{k, \chi}^{\text {off }}[n]}, \forall n \in \mathcal{N}_{1}, \forall k, \\
& L_{\mathrm{U}, k}^{\text {off }}[n]\left(t_{\mathrm{U}, k}^{\text {off }}[n]\right)=t_{\mathrm{U}, k}^{\text {off }}[n] \frac{\bar{L}_{\mathrm{U}, k, \chi+1}^{\text {off }}[n]}{t_{\mathrm{U}, k, \chi}^{\text {off }}[n]}, \forall n \in \mathcal{N}_{2}, \forall k, \\
& L_{\mathrm{U}, k}^{\text {down }}[n]\left(t_{\mathrm{U}, k}^{\text {down }}[n]\right)=t_{\mathrm{U}, k}^{\text {down }}[n] \frac{\bar{L}_{\mathrm{U}, k, \chi+1}^{\text {down }}[n]}{t_{\mathrm{U}, k, \chi}^{\text {down }}[n]}, \forall n \in \mathcal{N}_{3}, \forall k, \\
& L_{\mathrm{U}, k}[n]\left(t_{k}[n]\right)=t_{k}[n] f_{\mathrm{U}, k, \chi+1}[n] / C_{k}, \forall n \in \mathcal{N}_{2}, \forall k .
\end{aligned}
$$

Hence, the equivalent time allocation subproblem can be expressed as problem (P2) given below

$$
\begin{aligned}
(\mathrm{P} 2): \max _{\mathbf{t}} & \sum_{k=1}^{K} w_{k} \sum_{n=1}^{N} t_{k}^{\text {off }}[n] \frac{\bar{L}_{k, \chi+1}^{\text {off }}[n]}{t_{k, \chi}^{\text {off }}[n]} \\
\text { s.t. } & (26 \mathrm{~b})-(26 \mathrm{i}),(26 \mathrm{~m})-(26 \mathrm{r}),
\end{aligned}
$$

where the functions (53)-(56) are used in constraints (26d)-(26g), and the energy consumption expressions in (26h) and (26i) are referred to the forms in (6), (8a), (10a), (12a) and (14a) with the given $\mathbf{L}_{\chi+1}^{\text {loc }}, \mathbf{R}_{\chi+1}^{\text {res }}, \mathbf{P}_{\mathrm{U}, \chi+1}$ and $\mathbf{u}_{\chi}$. It can be verified that the sub-problem (P2) is a linear programming w.r.t. $\mathbf{t}$, and thus the optimal solution $\mathbf{t}_{\chi+1}$ can be readily solved by CVX [28]. The completed computation task-input bits at the UAV in the $(\chi+1)$-th iteration, i.e., $\left\{L_{\mathrm{U}, k, \chi+1}[n]\right\}$, can be finally achieved by substituting $\left\{t_{k, \chi+1}[n]\right\}$ into (56). 


\section{UAV Trajectory Design}

Here, the sub-problem for designing the UAV's trajectory $\mathbf{u}$ in the $(\chi+1)$-th iteration is considered, by assuming that the local computing task-input data size, the resource allocation for computation offloading, the time allocation, and the energy transmit power of the UAV are fixed as $\mathbf{L}_{\chi+1}^{\text {loc }}, \mathbf{R}_{\chi+1}^{\text {res }}, \mathbf{t}_{\chi+1}$, and $\mathbf{P}_{\mathrm{U}, \chi+1}$, respectively. Next, we update the computation task sizes related to offloading as the functions of $\mathbf{u}$ as

$$
\begin{aligned}
& L_{k}^{\text {off }}[n](\mathbf{u}[n])=t_{k, \chi+1}^{\text {off }}[n] B \log _{2}\left(1+\frac{P_{k, \chi+1}^{\text {off }}[n] h_{0} / N_{0}}{\left\|\mathbf{u}[n]-\mathbf{s}_{k}\right\|^{2}+H^{2}}\right), \\
& L_{\mathrm{U}, k}^{\text {off }}[n](\mathbf{u}[n])=t_{\mathrm{U}, k, \chi+1}^{\text {off }}[n] \log _{2}\left(1+\frac{P_{\mathrm{U}, k, \chi+1}^{\text {off }}[n] h_{0} / N_{0}}{\left\|\mathbf{u}[n]-\mathbf{s}_{0}\right\|^{2}+H^{2}}\right), \\
& L_{\mathrm{U}, k}^{\text {down }}[n](\mathbf{u}[n])=t_{\mathrm{U}, k, \chi+1}^{\text {down }}[n] \log _{2}\left(1+\frac{P_{\mathrm{U}, k, \chi+1}^{\text {down }}[n] h_{0} / N_{0}}{\left\|\mathbf{u}[n]-\mathbf{s}_{k}\right\|^{2}+H^{2}}\right) .
\end{aligned}
$$

Hence, the UAV trajectory design problem (P3) is written as

$$
\begin{aligned}
(\mathrm{P} 3): \max _{\mathbf{u}} & \sum_{k=1}^{K} w_{k} \sum_{n=1}^{N} L_{k}^{\text {off }}[n](\mathbf{u}[n]) \\
\text { s.t. } & (26 \mathrm{~d})-(26 \mathrm{i}),(26 \mathrm{~s}),(26 \mathrm{t}),
\end{aligned}
$$

where the functions (58)-(60) and $\left\{L_{\mathrm{U}, k, \chi+1}[n]\right\}$ are used in constraints (26d)-(26i), and the energy consumption expressions in (26h) and (26i) are referred to the forms in (6), (8b), (10b), (12b) and (14b) with the given $\mathbf{L}_{\chi+1}^{\text {loc }}, \mathbf{P}_{\mathrm{U}, \chi+1}$, and $\mathbf{t}_{\chi+1}$. Note that the objective function is not concave and the constraints (26d)-(26i) are not convex w.r.t u. To address this issue, we first equivalently re-express the problem (P3) as

$$
\begin{aligned}
(\mathrm{P} 3): \max _{\mathbf{u}, L_{\mathrm{W}}} & L_{\mathrm{W}} \\
\text { s.t. } & (26 \mathrm{~d})-(26 \mathrm{i}),(26 \mathrm{~s}),(26 \mathrm{t}), \\
& \sum_{k=1}^{K} w_{k} \sum_{n=1}^{N} L_{k}^{\text {off }}[n](\mathbf{u}[n]) \geq L_{\mathrm{W}},
\end{aligned}
$$

and then leverage the SCA algorithm to achieve an efficient solution of problem (P3).

In the $(m+1)$-th $(m=0,1,2, \ldots)$ iteration of the SCA algorithm, we first approximate the non-concave items $L_{k}^{\text {off }}[n](\mathbf{u}[n])$ in (26d), (62c), $L_{\mathrm{U}, k}^{\mathrm{off}}[n](\mathbf{u}[n])$ in (26e), (26f), $L_{\mathrm{U}, k}^{\text {down }}[n](\mathbf{u}[n])$ in (26g), $h_{k}[n](\mathbf{u}[n])$ in (26h), and $g_{\mathrm{AP}}[n](\mathbf{u}[n])$ in (26i), by their concave lower bounds, respectively denoted as $\widetilde{L}_{k}^{\text {off }}[n](\mathbf{u}[n]), \widetilde{L}_{\mathrm{U}, k}^{\text {off }}[n](\mathbf{u}[n]), \widetilde{L}_{\mathrm{U}, k}^{\text {down }}[n](\mathbf{u}[n]), \widetilde{h}_{k}[n](\mathbf{u}[n])$, and $\widetilde{g}_{\mathrm{AP}}[n](\mathbf{u}[n])$ as

$$
\begin{gathered}
L_{k}^{\text {off }}[n](\mathbf{u}[n]) \geq \widetilde{L}_{k}^{\text {off }}[n](\mathbf{u}[n])=t_{k, \chi+1}^{\text {off }}[n] B\left\{\log _{2}\left(1+\frac{P_{k, \chi+1}^{\text {off }}[n] h_{0} / N_{0}}{d_{k}^{2}\left(\mathbf{u}_{m}[n]\right)}\right)\right. \\
\left.-\frac{P_{k, \chi+1}^{\text {off }}[n] h_{0}}{N_{0} \ln 2} \frac{\left\|\mathbf{u}[n]-\mathbf{s}_{k}\right\|^{2}-\left\|\mathbf{u}_{m}[n]-\mathbf{s}_{k}\right\|^{2}}{d_{k}^{2}\left(\mathbf{u}_{m}[n]\right)\left(d_{k}^{2}\left(\mathbf{u}_{m}[n]\right)+P_{k, \chi+1}^{\text {off }}[n] h_{0} / N_{0}\right)}\right\} \\
L_{\mathrm{U}, k}^{\mathrm{off}}[n](\mathbf{u}[n]) \geq \widetilde{L}_{\mathrm{U}, k}^{\mathrm{off}}[n](\mathbf{u}[n])=t_{\mathrm{U}, k, \chi+1}^{\mathrm{off}}[n] B\left\{\log _{2}\left(1+\frac{P_{\mathrm{U}, k, \chi+1}^{\mathrm{off}}[n] h_{0} / N_{0}}{d_{\mathrm{AP}}^{2}\left(\mathbf{u}_{m}[n]\right)}\right)\right.
\end{gathered}
$$




$$
\begin{gathered}
\left.-\frac{P_{\mathrm{U}, k, \chi+1}^{\mathrm{off}}[n] h_{0}}{N_{0} \ln 2} \frac{\left\|\mathbf{u}[n]-\mathbf{s}_{0}\right\|^{2}-\left\|\mathbf{u}_{m}[n]-\mathbf{s}_{0}\right\|^{2}}{d_{\mathrm{AP}}^{2}\left(\mathbf{u}_{m}[n]\right)\left(d_{\mathrm{AP}}^{2}\left(\mathbf{u}_{m}[n]\right)+P_{\mathrm{U}, k, \chi+1}^{\mathrm{off}}[n] h_{0} / N_{0}\right)}\right\} \\
L_{\mathrm{U}, k}^{\text {down }}[n](\mathbf{u}[n]) \geq \widetilde{L}_{\mathrm{U}, k}^{\mathrm{down}}[n](\mathbf{u}[n])=t_{\mathrm{U}, k, \chi+1}^{\mathrm{down}}[n] B\left\{\log _{2}\left(1+\frac{P_{\mathrm{U}, k, \chi+1}^{\mathrm{down}}[n] h_{0} / N_{0}}{d_{k}^{2}\left(\mathbf{u}_{m}[n]\right)}\right)\right. \\
\left.-\frac{P_{\mathrm{U}, k, \chi+1}^{\mathrm{down}}[n] h_{0}}{N_{0} \ln 2} \frac{\left\|\mathbf{u}[n]-\mathbf{s}_{k}\right\|^{2}-\left\|\mathbf{u}_{m}[n]-\mathbf{s}_{k}\right\|^{2}}{d_{k}^{2}\left(\mathbf{u}_{m}[n]\right)\left(d_{k}^{2}\left(\mathbf{u}_{m}[n]\right)+P_{\mathrm{U}, k, \chi+1}^{\text {down }}[n] h_{0} / N_{0}\right)}\right\} \\
h_{\mathrm{AP}}[n](\mathbf{u}[n]) \geq \widetilde{h}_{k}[n](\mathbf{u}[n])=h_{0} \frac{d_{k}^{2}\left(\mathbf{u}_{m}[n]\right)+\left\|\mathbf{u}_{m}[n]-\mathbf{s}_{k}\right\|^{2}-\left\|\mathbf{u}[n]-\mathbf{s}_{k}\right\|^{2}}{d_{k}^{4}\left(\mathbf{u}_{m}[n]\right)}, \\
\widetilde{g}_{\mathrm{AP}}[n](\mathbf{u}[n])=g_{\mathrm{AP}}[n]\left(\mathbf{u}_{m}[n]\right)+g_{\mathrm{AP}}^{\prime}[n]\left(\left\|\mathbf{u}_{m}[n]-\mathbf{s}_{0}\right\|^{2}\right)\left(\left\|\mathbf{u}[n]-\mathbf{s}_{0}\right\|^{2}-\left\|\mathbf{u}_{m}[n]-\mathbf{s}_{0}\right\|^{2}\right),
\end{gathered}
$$

which are obtained using the first-order Taylor expansion around the optimized trajectory at the $m$-th iteration, denoted as $\mathbf{u}_{m}[n]$, w.r.t. the convex item $\left\|\mathbf{u}[n]-\mathbf{s}_{i}\right\|^{2}, i=0$ or $k$. Similarly, the non-convex items $L_{k}^{\text {off }}[n](\mathbf{u}[n])$ in (26f), $L_{\mathrm{U}, k}^{\text {off }}[n](\mathbf{u}[n])$ in $(26 \mathrm{~d}),(26 \mathrm{~g})$, and $L_{\mathrm{U}, k}^{\text {down }}[n](\mathbf{u}[n])$ in (26e), are approximated by their convex upper bounds, respectively denoted as $\widehat{L}_{k}^{\text {off }}[n](\mathbf{u}[n])$, $\widehat{L}_{\mathrm{U}, k}^{\text {off }}[n](\mathbf{u}[n])$ and $\widehat{L}_{\mathrm{U}, k}^{\text {down }}[n](\mathbf{u}[n])$ given below

$$
\begin{gathered}
L_{k}^{\text {off }}[n](\mathbf{u}[n]) \leq \widehat{L}_{k}^{\text {off }}[n](\mathbf{u}[n])=t_{k, \chi+1}^{\text {off }}[n] B \log _{2}\left(1+\frac{P_{k, \chi+1}^{\text {off }}[n] h_{0} / N_{0}}{d_{k}^{2}\left(\mathbf{u}_{m}[n]\right)+2\left(\mathbf{u}_{m}[n]-\mathbf{s}_{k}\right)^{T}\left(\mathbf{u}[n]-\mathbf{u}_{m}[n]\right)}\right), \\
L_{\mathrm{U}, k}^{\text {off }}[n](\mathbf{u}[n]) \leq \widehat{L}_{\mathrm{U}, k}^{\text {off }}[n](\mathbf{u}[n])=t_{\mathrm{U}, k, \chi+1}^{\text {off }}[n] B \log _{2}\left(1+\frac{P_{\mathrm{U}, k, \chi+1}^{\text {off }}[n] h_{0} / N_{0}}{d_{\mathrm{AP}}^{2}\left(\mathbf{u}_{m}[n]\right)+2\left(\mathbf{u}_{m}[n]-\mathbf{s}_{0}\right)^{T}\left(\mathbf{u}[n]-\mathbf{u}_{m}[n]\right)}\right), \\
L_{\mathrm{U}, k}^{\text {down }}[n](\mathbf{u}[n]) \leq \widehat{L}_{\mathrm{U}, k}^{\text {down }}[n](\mathbf{u}[n])=t_{\mathrm{U}, k, \chi+1}^{\text {down }}[n] B \log _{2}\left(1+\frac{P_{\mathrm{U}, k, \chi+1}^{\text {down }}[n] h_{0} / N_{0}}{d_{k}^{2}\left(\mathbf{u}_{m}[n]\right)+2\left(\mathbf{u}_{m}[n]-\mathbf{s}_{k}\right)^{T}\left(\mathbf{u}[n]-\mathbf{u}_{m}[n]\right)}\right),
\end{gathered}
$$

which are obtained based on the fact that $\left\|\mathbf{u}[n]-\mathbf{s}_{i}\right\|^{2} \geq\left\|\mathbf{u}_{m}[n]-\mathbf{s}_{i}\right\|^{2}+2\left(\mathbf{u}_{m}[n]-\mathbf{s}_{i}\right)^{T}(\mathbf{u}[n]-$ $\left.\mathbf{u}_{m}[n]\right), i=0$ or $k$. To properly deal with the non-linear equality constraints in (26f) and (26g) in problem $(\mathrm{P} 3)$, we relax them as the following convex inequality constraints

$$
\begin{aligned}
& \sum_{n=2}^{N-1}\left(\widetilde{L}_{\mathrm{U}, k}[n]+\widetilde{L}_{\mathrm{U}, k}^{\mathrm{off}}[n]\right)-\sum_{n=1}^{N-2} \widehat{L}_{k}^{\mathrm{off}}[n] \geq-\delta, \quad \forall k, \\
& \sum_{n=3}^{N} \widetilde{L}_{\mathrm{U}, k}^{\text {down }}[n]-O_{k} \sum_{n=2}^{N-1}\left(\widehat{L}_{\mathrm{U}, k}[n]+\widehat{L}_{\mathrm{U}, k}^{\mathrm{off}}[n]\right) \geq-\delta, \forall k,
\end{aligned}
$$

where $\delta>0$ is a preset tolerant threshold.

It is further noted that the UAV's propulsion energy consumption $E_{\mathrm{U}}^{\mathrm{prop}}[n]$ defined in (21) is not a convex function of $\mathbf{u}$, which makes the energy causality constraints in (26i) non-convex. In order to deal with this issue, we first define a convex upper bound of $E_{\mathrm{U}}^{\mathrm{prop}}[n]$ as

$$
\widehat{E}_{\mathrm{U}}^{\text {prop }}[n]=\tau\left(\zeta_{1}\|\mathbf{v}[n]\|^{3}+\frac{\zeta_{2}}{v[n]}\right), \forall n,
$$

by introducing an auxiliary variable $v[n]$ with a constraint $\|\mathbf{v}[n]\| \geq v[n]$, i.e., $\|\mathbf{u}[n]-\mathbf{u}[n-1]\|^{2} \geq$ $v^{2}[n] \tau^{2}$. In the $m$-th iteration of the SCA method, $\|\mathbf{u}[n]-\mathbf{u}[n-1]\|^{2}$ is then approximated as 
its linear lower bound by using the first-order Taylor expansion at a local point $\mathbf{u}_{m}$. In this way, the non-convex additional constraint can be approximated by its convex counterpart as

$$
v^{2}[n] \tau^{2}-2\left(\mathbf{u}_{m}[n]-\mathbf{u}_{m}[n-1]\right)^{T}(\mathbf{u}[n]-\mathbf{u}[n-1])+\left\|\mathbf{u}_{m}[n]-\mathbf{u}_{m}[n-1]\right\|^{2} \leq 0, \forall n .
$$

With the analysis above, we can formulate an approximated version of (P3) with $\left\{\widehat{L}_{\mathrm{U}, k}^{\text {off }}[n](\mathbf{u}[n])\right\}$ and $\left\{\widetilde{L}_{k}^{\text {off }}[n](\mathbf{u}[n])\right\}$ in $(26 \mathrm{~d}),\left\{\widehat{L}_{\mathrm{U}, k}^{\text {down }}[n](\mathbf{u}[n])\right\}$ and $\left\{\widetilde{L}_{\mathrm{U}, k}^{\text {off }}[n](\mathbf{u}[n])\right\}$ in $(26 \mathrm{e}),\left\{\widetilde{h}_{k}[n](\mathbf{u}[n])\right\}$ in (26h), $\left\{\widehat{E}_{\mathrm{U}}^{\text {prop }}[n]\right\}$ and $\left\{\widetilde{g}_{\mathrm{AP}}[n](\mathbf{u}[n])\right\}$ in (26i), $\left\{\widetilde{L}_{k}^{\text {off }}[n](\mathbf{u}[n])\right\}$ in (62c), and with the relaxed convex constraints (71), (72) and (74), in the $(m+1)$-th iteration of the SCA method. This approximated problem are convex w.r.t. the UAV's location vector u. However, the UAV's maximum speed constraint (26t) shows that the UAV's locations in different slots are coupled with each other, and thus it is difficult to obtain a closed-form solution of $\mathbf{u}$. Hence, we resort to CVX [28] to solve (P3) using SCA, and finally we can obtain the UAV's trajectory in the $(\chi+1)$-th iteration, i.e., $\mathbf{u}_{\chi+1}$.

After obtaining $\mathbf{L}_{\chi+1}^{\text {loc }}, \mathbf{R}_{\chi+1}^{\text {res }}, \mathbf{P}_{\mathrm{U}, \chi+1}, \mathbf{t}_{\chi+1}$, and $\mathbf{u}_{\chi+1}$, we can finally achieve the task allocation parameters in the $(\chi+1)$-th iteration, i.e., $\mathbf{L}_{\chi+1}=\left\{L_{k, \chi+1}^{\text {loc }}[n], L_{k, \chi+1}^{\text {off }}[n], L_{\mathrm{U}, k, \chi+1}[n]\right.$, $\left.L_{\mathrm{U}, k, \chi+1}^{\mathrm{off}}[n], L_{\mathrm{U}, k, \chi+1}^{\text {down }}[n]\right\}_{k \in \mathcal{K}, n \in \mathcal{N}}$. Here, the task parameters $L_{k, \chi+1}^{\mathrm{off}}[n], L_{\mathrm{U}, k, \chi+1}^{\mathrm{off}}[n]$ and $L_{\mathrm{U}, k, \chi+1}^{\text {down }}[n]$ are obtained by substituting $\mathbf{u}_{\chi+1}$ into (58), (59) and (60), respectively. Hence, the objective value of (26), i.e., the value of the UEs' WSCTB, in the $(\chi+1)$-th iteration can be found as

$$
L_{\chi+1}=\sum_{k=1}^{K} w_{k}\left(\sum_{n=1}^{N} L_{k, \chi+1}^{\mathrm{loc}}[n]+\sum_{n=1}^{N-2} L_{k, \chi+1}^{\mathrm{off}}[n]\right) .
$$

\section{Algorithm, Convergence, and Complexity}

The three-step block coordinate descending algorithm is summarized in Algorithm 1, where the WSCTB maximization problem (26) is solved in an iterative fashion with guaranteed convergence. In the first iteration of the algorithm, with a given initial solution of $\mathbf{t}$ and $\mathbf{u}$, the WSCTB can be obtained after optimizing $\mathbf{L}$ and $\mathbf{P}_{\mathrm{U}}$ through solving the computation task and the UAV's WPT power allocation problem (P1), which is convex and can be optimally solved through the proposed Lagrange method (step 1) with guaranteed convergence [33]; then the WSCTB will increase after obtaining a better solution of $\mathbf{t}$ through optimally solving the linear time allocation problem (P2) with CVX (step 2); and the WSCTB will further increase after obtaining a better solution of $\mathbf{u}$ by solving the UAV trajectory design problem (P3) through the proposed SCA method (step 3). Although (P3) is non-convex, we can always find a better solution than the initial one based on the SCA method also with guaranteed convergence [33]. Then we can obtain the 
$\overline{\text { Algorithm } 1 \text { Three-Step Block Coordinate Descending Algorithm for Solving the WSCTB }}$ Maximization Problem (26)

1: Set $T, N, K, B, h_{0}, N_{0}, P_{0}, H, V_{\max }, A, \vartheta, D, \beta, \alpha, \mathbf{s}_{0}, \mathbf{u}_{\mathrm{I}}, \mathbf{u}_{\mathrm{F}}, \eta_{\mathrm{U}}, \kappa_{\mathrm{U}}, \zeta_{1}, \zeta_{2},\left\{\mathbf{s}_{k}, w_{k}, C_{k}, O_{k}, \eta_{k}, \kappa_{k}\right\}_{k \in \mathcal{K}}$, the iterative steps of the subgradient method: $\left\{\varepsilon_{j}^{(\gamma)}\right\},\left\{\varepsilon_{j}^{(\mu)}\right\},\left\{\varepsilon_{j}^{(\xi)}\right\},\left\{\varepsilon_{j}^{(\rho)}\right\}$, and the tolerant thresholds $\epsilon$, $\varepsilon$;

2: Initialize the iteration index $\chi=1$ and $\mathbf{t}_{1}, \mathbf{u}_{1}$;

\section{3: Repeat 1}

4: $\quad$ Step 1: Initialize $j=1$, as well as $\gamma_{1}, \boldsymbol{\mu}_{1}, \boldsymbol{\xi}_{1}$ and $\boldsymbol{\rho}_{1}$;

5: $\quad$ Repeat 1.1

6: $\quad$ a) Obtain $\boldsymbol{\lambda}_{j}, \boldsymbol{\nu}_{j}$ with $\boldsymbol{\gamma}_{j}, \boldsymbol{\mu}_{j}, \boldsymbol{\xi}_{j}, \boldsymbol{\rho}_{j}$ using Lemma 2;

b) Obtain $\overline{\mathbf{L}}_{\chi, j}=\left\{L_{k, j}^{\text {loc }}[n], \bar{L}_{k, j}^{\text {off }}[n], \bar{L}_{\mathrm{U}, k, j}[n], \bar{L}_{\mathrm{U}, k, j}^{\mathrm{off}}[n], \bar{L}_{\mathrm{U}, k, j}^{\text {down }}[n]\right\}, \mathbf{P}_{\mathrm{U}, \chi, j}=\left\{P_{\mathrm{U}, j}[n]\right\}$ from Theorem 1 with $\gamma_{j}, \boldsymbol{\mu}_{j}, \boldsymbol{\xi}_{j}, \boldsymbol{\rho}_{j}, \boldsymbol{\lambda}_{j}, \boldsymbol{\nu}_{j}$ and $\mathbf{t}_{\chi}, \mathbf{u}_{\chi}$;

c) Calculate the WSCTB, denoted as $L_{j}$, by substituting $\overline{\mathbf{L}}_{\chi, j}$ into the objective function of problem (P1);

d) Update $\boldsymbol{\gamma}_{j+1}, \boldsymbol{\mu}_{j+1}, \boldsymbol{\xi}_{j+1}, \boldsymbol{\rho}_{j+1}$ through Lemma 1;

e) $j=j+1$;

7: $\quad$ End Repeat 1.1 until convergence, i.e., $\left|L_{j}-L_{j-1}\right|<\epsilon(j>1)$, and obtain $\overline{\mathbf{L}}_{\chi+1}=\overline{\mathbf{L}}_{\chi, j}$ (i.e., $\mathbf{L}_{\chi+1}^{\text {loc }}$ and $\mathbf{R}_{\chi+1}^{\text {res }}$ ), $\mathbf{P}_{\mathrm{U}, \chi+1}=\mathbf{P}_{\mathrm{U}, \chi, j}$ with the obtained $\gamma_{\chi}, \boldsymbol{\mu}_{\chi}, \boldsymbol{\xi}_{\chi}, \boldsymbol{\rho}_{\chi}$, and $\boldsymbol{\lambda}_{\chi}, \boldsymbol{\nu}_{\chi}$ at the convergence;

8: $\quad$ Step 2: Solve the problem (P2) by CVX with the given $\mathbf{L}_{\chi+1}^{\text {loc }}, \mathbf{R}_{\chi+1}^{\text {res }}, \mathbf{P}_{\mathrm{U}, \chi+1}$ and $\mathbf{u}_{\chi}$; Then obtain the optimal solution, i.e., $\mathbf{t}_{\chi+1}$, and $\left\{L_{\mathrm{U}, k, \chi+1}[n]\right\}$.

9: Step 3: Solve the problem of (P3) through the SCA method with the help of CVX and the given $\mathbf{L}_{\chi+1}^{\text {loc }}$, $\mathbf{R}_{\chi+1}^{\text {res }}, \mathbf{P}_{\mathrm{U}, \chi+1}$ and $\mathbf{t}_{\chi+1}$; Then obtain the final solution, i.e., $\mathbf{u}_{\chi+1}$;

10: Obtain the task allocation at the $(\chi+1)$-th iteration, i.e., $\mathbf{L}_{\chi+1}$, with the obtained $\mathbf{L}_{\chi+1}^{\text {loc }}, \mathbf{R}_{\chi+1}^{\text {res }}, \mathbf{P}_{\mathrm{U}, \chi+1}, \mathbf{t}_{\chi+1}$, and $\mathbf{u}_{\chi+1}$; Then calculate the WSCTB through (75).

11: $\chi=\chi+1$;

12: End Repeat 1 until convergence, i.e., $\left|L_{\chi}-L_{\chi-1}\right|<\varepsilon(\chi>2)$, and obtain the maximum WSCTB $L_{\chi}$ with the solution $\mathbf{L}^{*}=\mathbf{L}_{\chi}, \mathbf{P}_{\mathrm{U}}^{*}=\mathbf{P}_{\mathrm{U}, \chi}, \mathbf{t}^{*}=\mathbf{t}_{\chi}, \mathbf{u}^{*}=\mathbf{u}_{\chi}$;

corresponding WSCTB, i.e., $L_{\chi=2}$ with the finally updated task allocation parameters in $\mathbf{L}_{\chi=2}$. In the subsequent iterations, the WSCTB will continue increasing in the same way described for the first iteration, which means that the objective function of the WSCTB maximization problem (26) monotonically increases with the iteration index $\chi$ by optimizing $\mathbf{L}, \mathbf{P}_{\mathrm{U}}, \mathbf{t}$ and $\mathbf{u}$ alternatingly. Besides, the achieved WSCTB $L_{\chi}$ is upper bounded due to the limited system energy $P_{0} T$, further ensuring the convergence of the algorithm.

The proposed three-step block coordinate descending algorithm is easy to implement with 
acceptable complexity. In Step 1, the complexity mainly comes from the subgradient method for obtaining $\left\{\gamma_{k, n}\right\},\left\{\mu_{k, n}\right\},\left\{\xi_{k, n}\right\},\left\{\rho_{n}\right\}$, and the bi-section searches of $\left\{\sum_{n=1}^{N-2} \bar{L}_{k}^{\text {off }}[n]\right\},\left\{\lambda_{k}\right\}$, $\left\{\nu_{k}\right\}$ in each iteration. Let $\theta_{\text {sub }}>0$, and $\theta_{L}, \theta_{\lambda}, \theta_{\nu}>0$ denote the computational accuracies of the subgradient method and the bisectional searches for $\left\{\sum_{n=1}^{N-2} \bar{L}_{k}^{\text {off }}[n]\right\},\left\{\lambda_{k}\right\},\left\{\nu_{k}\right\}$. Thus, the corresponding complexity for Step 1 can be calculated as $\mathcal{O}\left(1 / \theta_{\text {sub }}^{2}+K \log _{2}\left(1 / \theta_{L}\right)\left(\log _{2}\left(1 / \theta_{\lambda}\right)+\right.\right.$ $\left.\log _{2}\left(1 / \theta_{\nu}\right)\right)$ ). Step 2 focuses on solving the time allocation problem (P2), which is a linear programming and can be solved by CVX with an acceptable complexity. In Step 3, the approximated problem of the UAV's trajectory design problem (P3) in each iteration of the SCA method, can also be efficiently solved by CVX, which also has an acceptable complexity.

TABLE I

SIMULATION PARAMETERS

\begin{tabular}{|l|l|l|}
\hline Parameter & Symbol & Value \\
\hline The total task completion time & $T$ & 5 seconds \\
\hline Number of time slots & $N$ & 50 \\
\hline Number of ground UEs & $K$ & 4 and 8 \\
\hline The total system bandwidth & $B$ & $400 \mathrm{MHz}$ \\
\hline The channel power gain at a reference distance of $d_{0}=1 \mathrm{~m}$ & $h_{0}$ & $-30 \mathrm{~dB}$ \\
\hline The noise power & $N_{0}$ & $-60 \mathrm{dBm}$ \\
\hline The energy transmit power of the AP & $P_{0}$ & $60 \mathrm{dBm}$ \\
\hline The fixed altitude of the UAV & $H$ & $10 \mathrm{~m}$ \\
\hline The maximum available speed of the UAV & $V_{\text {max }}$ & $20 \mathrm{~m} / \mathrm{s}$ \\
\hline The area of the laser receiver's telescope or collection & $A$ & $10^{-2} \mathrm{~m}^{2}$ \\
\hline The size of the initial laser beam & $D$ & $0.05 \mathrm{~m}$ \\
\hline The combined transmission receiver optical efficiency & $\vartheta$ & 0.2 \\
\hline The angular spread & $\beta$ & $3.4 * 10^{-5}$ \\
\hline The attenuation coefficient of the channel medium & $\alpha$ & $10^{-6} / \mathrm{m}$ \\
\hline The energy conversion efficiencies & $\eta_{\mathrm{U}}, \eta_{k}, \forall k$ & 0.8 \\
\hline The effective switched capacitance of the UAV and UEs & $\kappa_{\mathrm{U}}, \kappa_{k}, \forall k$ & $10^{-28}$ \\
\hline The UAV's propulsion energy consumption related parameters & $\left(\zeta_{1}, \zeta_{2}\right)$ & $(0.00614,15.976)$ \\
\hline Required CPU cycles per bit & $C_{k}, \forall k$ & $100 \mathrm{cycles} / \mathrm{bit}$ \\
\hline UEs' task size ratio of output data to input data & $O_{k}, \forall k$ & 0.5 \\
\hline The tolerant thresholds & $\epsilon, \varepsilon$ & $10^{-4}$ \\
\hline
\end{tabular}

\section{Simulation Results}

Here, simulation results are given to evaluate the performance of the proposed algorithm by comparing with some benchmark schemes. The basic simulation parameters are listed in Table I. ${ }^{8}$

\section{A. Trajectory of the $U A V$}

In this subsection, numerical results for UAV's trajectory are given in Figs. 3-5, to shed light on the impact of the UEs' weight vector $(\mathbf{w})$, the AP's location $\left(\mathbf{s}_{0}\right)$, and the randomness of UEs'

${ }^{8}$ The parameters related to propulsion energy consumption of fixed-wing UAV, i.e., $\zeta_{1}, \zeta_{2}$ in (21), are set based on references [21,29], while the parameters of laser charging model, i.e., $A, \vartheta, D, \beta, \alpha$ in (18), are set based on references [25, 26]. 
and AP's locations as well as UEs' weight allocation. In Figs. 3 and 4, 4 UEs are served in a $10 \mathrm{~m} \times 10 \mathrm{~m}$ central area with given locations $\left[\mathbf{s}_{1}, \mathbf{s}_{2}, \mathbf{s}_{3}, \mathbf{s}_{4}\right]=[(5,5),(-5,5),(-5,-5),(5,-5)]$ and UAV's initial and final locations $\mathbf{u}_{\mathrm{I}}=(-5,-5), \mathbf{u}_{\mathrm{F}}=(5,-5)$. The cases of 6 UEs, 8 UEs, 10 UEs and 12 UEs being served in a $20 \mathrm{~m} \times 20 \mathrm{~m}$ central area with random locations and weights are considered in Fig. 5, where $\mathbf{u}_{\mathrm{I}}=(-10,-10), \mathbf{u}_{\mathrm{F}}=(10,-10)$.
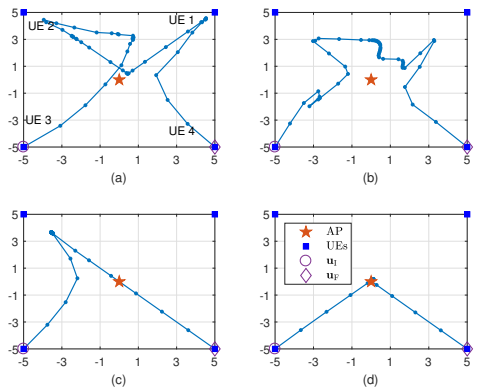

Fig. 3. The UAV's trajectories for serving 4 UEs in situations with different UEs' weight vectors: $\mathbf{w}=[0.5,0.3,0.1,0.1]$ for (a), $\mathbf{w}=[0.3,0.4,0.2,0.1]$ for $(b), \mathbf{w}=[0.1,0.7,0.1,0.1]$ for (c), and $\mathbf{w}=[0.25,0.25,0.25,0.25]$ for $(\mathrm{d})$.
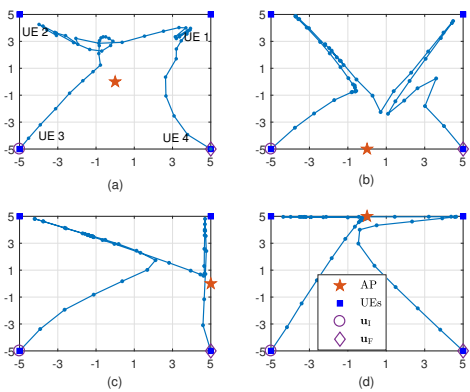

Fig. 4. The UAV's trajectories for serving 4 UEs in situations with different AP's location $\mathbf{s}_{0}: \mathbf{s}_{0}=(0,0)$ for $(\mathrm{a}), \mathbf{s}_{0}=(0,-5)$ for $(\mathrm{b}), \mathbf{s}_{0}=(5,0)$ for $(\mathrm{c})$, and $\mathbf{s}_{0}=(0,5)$ for $(\mathrm{d})$.

Fig. 3 depicts the UAV's trajectories in the scenarios with different UEs' weight vectors, where the AP is fixed at the origin, i.e., $\mathbf{s}_{0}=(0,0)$. It can be seen that the UAV tends to fly close to the UEs with larger weight values, such as UE 1 and UE 2 in (a), (b), and UE 2 in (c). This coincides with the intuition that the UEs with higher priorities should have higher qualities considering both the system setting and resource scheduling. However, it is interesting to see that the UAV does not simply head to these UEs one by one directly, but always try to find a way being close to the AP. Especially at the several initial time slots, the UAV almost flies directly to the AP. This is due to the fact that the UAV can harvest and store more energy when getting closer to the AP, and the stored energy is essential to support the UAV's further operations, such as broadcasting energy, offloading UEs' task-input bits to the AP, downloading the UEs' task-output bits to the corresponding UEs as well as flying. Besides, being close to the AP can save the UAV's energy used for further offloading UEs' tasks to the AP.

To further show the effects of AP's location on the UAV's trajectory, we investigate the UAV's trajectories with different AP's locations in Fig. 4 with $\mathbf{w}=[0.4,0.3,0.2,0.1]$ for 4 UEs. This figure clearly shows that the UAV's trajectories highly depend on the AP's locations. No matter 

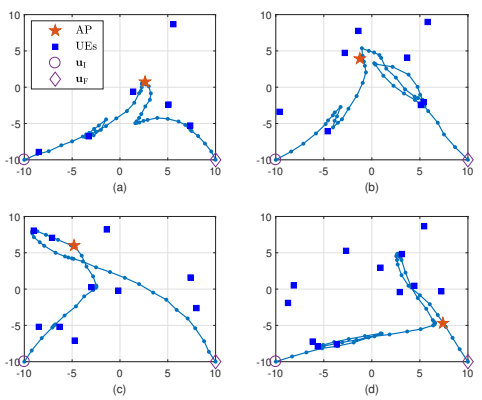

Fig. 5. The UAV's trajectories in situations with randomly distributed AP, UEs and random weights for UEs from left to right: (a) 6 UEs with $\mathbf{w}=[0.1901,0.3245,0.0043,0.1634,0.0465,0.2712]$; (b) 8 UEs with $\mathbf{w}=[0.1109,0.2148,0.1032,0.1022$, 0.1156, 0.0565, 0.2113, 0.0855]; (c) 10 UEs with $\mathbf{w}=[0.2026,0.0322,0.1050,0.1091,0.0356,0.2069,0.0475,0.0883,0.1437$, 0.0291]; (d) 12 UEs with $\mathbf{w}=[0.0605,0.0715,0.0939,0.0148,0.1267,0.0847,0.0733,0.0862,0.1612,0.1340,0.0401,0.0531]$.

where the AP is, the UAV's trajectories look like a radiation pattern centred at the AP and radiated to the UEs especially those with larger weight values. The reason behind this phenomenon is that the AP plays two essential roles in determining UAV's trajectory, one for being the original energy source and another for being a powerful MEC server. These two roles incentivize the UAV to find a way to get close to the AP, as described in the analysis of Fig. 3.

In Fig. 5, we show the trajectories of 4 more practical scenarios with 6, 8, 10 and 12 UEs, where the locations of AP and UEs are randomly distributed in a $20 \mathrm{~m} \times 20 \mathrm{~m}$ central area. Also, the UEs' normalized weights are randomly chosen. Similar observations can be obtained from Figs. 3 and 4. In addition, the results indicate that In order to achieve a good system performance for maximizing the WSCTB of UEs, the UAV's trajectory should be properly designed by considering the trade-off between its distances to the AP and UEs, especially the UEs with larger weight values. Getting close to the the AP is beneficial to energy harvesting and reducing the energy consumption for further computation offloading to the AP. In contrast, being close to the UEs with larger weight values can help reduce the UEs' energy consumed for computation offloading to the UAV and the UAV's energy used for energy broadcasting and task-output data downloading to the UEs. The saved energy of the UAV and the UEs can be utilized to complete more task-input data, resulting in improvement of the WSCTB of UEs. It is interesting to note that the trade-off between the UAV's distances to the AP and UEs may lead to back-and-forth tracks of the UAV between the AP and UEs, which is clearly demonstrated in Figs. 3(a), (b), Figs. 4(a), (b) and Figs. 5(a), (b), (d). 


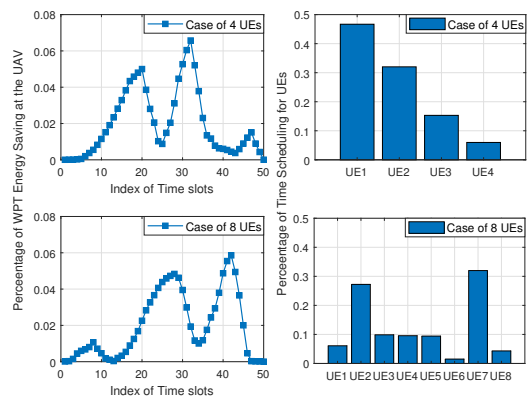

Fig. 6. The percentages of WPT energy saving at the UAV and the time scheduling for UEs.

In Fig. 6, we study the relationships between the UAV's trajectories and the UAV's energy saving as well as the UEs' time scheduling by examining the percentage of UAV's WPT energy saving w.r.t. the index of the time slots and the percentage of UEs' time scheduling in the whole task completion time T. Two cases of 4 UEs and 8 UEs as in Fig. 4(a) and Fig. 5(b) are considered. Referring to the trajectories shown in Fig. 4(a) and Fig. 5(b), it can be seen that the UAV tends to harvest and store more energy when flying close to the AP, leading to two peaks in the energy saving curves. In addition, the UAV's energy saving decreases dramatically when flying close to the UE 2, UE 1 in Fig. 4(a), and UE 2, UE 7 in Fig. 5(b), indicating that the UAV consumes more energy for energy broadcasting and data downloading when flying close to the UEs especially those with larger wights in order to increase the energy harvesting efficiency and improve the system performance. From the histograms of UEs' time scheduling, we can observe that the time scheduling is highly related to the UEs' weight values but not exactly to the same ratio. Specifically, the UEs with large weights tend to pull the UAV close to them in order to increase the WSCTB, and thus more time will be scheduled to these UEs.

\section{B. Performance Improvement}

In this section, the performance improvement of the proposed algorithm, i.e., the WSCTB, is verified by simulation results in comparison with several benchmark schemes. The benchmarks include the 'Offloading Only' scheme where computation offloading is the only way for computing without considering local computing at UEs; the 'UAV as Relay Only' scheme where the UAV acts as an information and an energy relay but not an MEC server; the 'Equal Time Allocation' scheme with fixed equal time allocation for all UEs and their active operations; and the 'Semi-circle Trajectory' scheme where the UAV flies through a fixed semi-circle trajectory with a constant speed (the semi-circle uses the line segment between UAV's initial and final 
locations as the diameter and goes through the origin). In Figs. 7-9, we also show the results in two cases, one for 4 UEs as in Fig. 4(a) and one for 8 UEs as in Fig. 5(b).

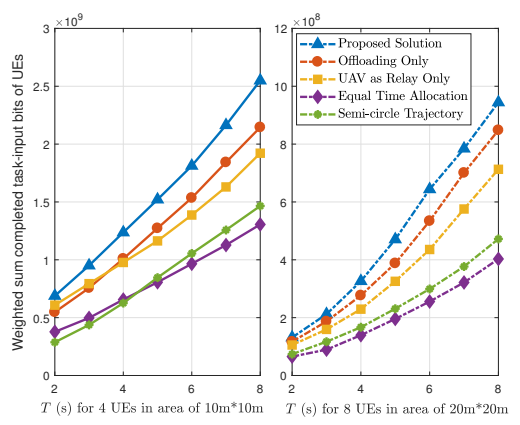

Fig. 7. The weighted sum completed task-input bits (WSCTB) of UEs versus the total task completion time $T$.

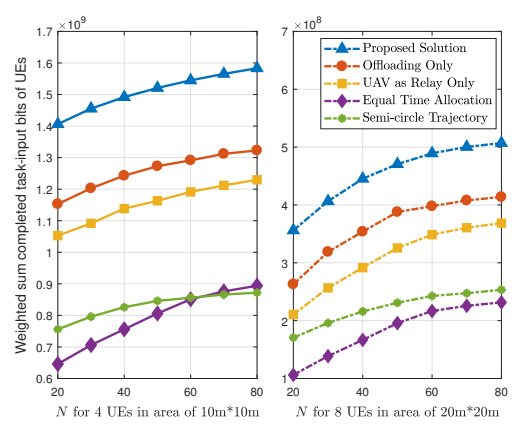

Fig. 8. The weighted sum completed task-input bits (WSCTB) of UEs versus the number of time slots $N$.

In Fig. 7, the UEs' WSCTB results are plotted against the task completion time $T$. As we can see, the WSCTBs of all the schemes increase with $T$, while the proposed solution outperforms all the other benchmark schemes considerably in both cases. Also, the gaps between the proposed solution and the other schemes get larger when $T$ is larger, which means that the advantages of the proposed algorithm in improving the UEs' WSCTB become more obvious when the system resource is more abundant. This is realized by properly leveraging all computing resources in the system, through jointly optimizing the computation task allocation, time allocation, and the UAV's WPT power and its trajectory. Note that the 'Equal Time Allocation' and 'Semi-circle Trajectory' schemes are obviously worse than the other schemes, reflecting the great effects of optimizing the time allocation and the UAV's trajectory.

Fig. 8 shows the WSCTBs of all the schemes w.r.t. the number of time slots $N$, which further indicates that the proposed solution can achieve better performance in comparison with the other benchmarks. We see that the performance of all the schemes enhances as $N$ increases, and this owes to the fact that the precision of the algorithm gradually improves with an increasing of $N$ (smaller time slots with larger $N$ ), but at the cost of increasing the complexity of the algorithm. Hence, there is a trade-off between the system performance and the algorithm complexity through $N$. Particularly, the flexibility of the UAV strengthens as $N$ increases, and thus the negative effects of fixing the UAV's trajectory become more obvious, supported by the results that the WSCTBs of the 'Semi-circle Trajectory' scheme increase slower than the other schemes and it is even worse than the 'Equal Time Allocation' scheme in the case of 4 UEs when $N$ is large. In 
addition, it is easy to note that the increments of all the schemes decrease and the corresponding WSCTBs gradually saturate as $N$ increases, due to the limitation of the system resources.

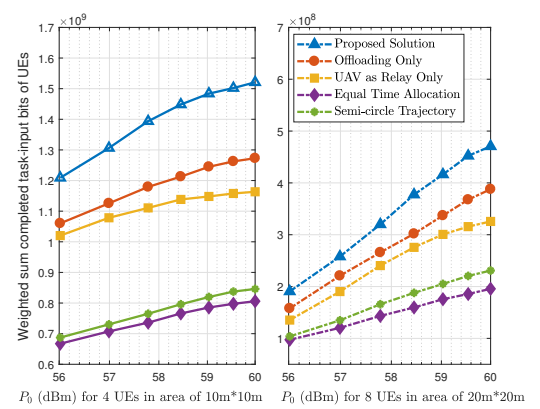

Fig. 9. The weighted sum completed task-input bits (WSCTB) of UEs versus the AP's laser energy transmit power $P_{0}$.

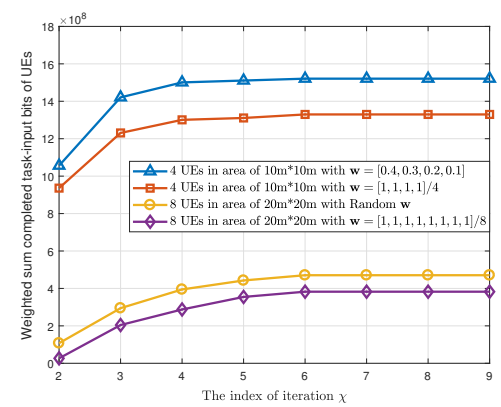

Fig. 10. The weighted sum completed task-input bits (WSCTB) of UEs versus the index of iteration $\chi$.

The effects of the AP's laser energy transmit power, i.e., $P_{0}$, on the WSCTB are investigated by the results in Fig. 9. As above, the proposed solution performs better than all the baseline schemes. This figure further shows that the WSCTBs of all the schemes increase with $P_{0}$, which coincides with the intuition. We can also see that the corresponding increasing rates of the WSCTBs decrease versus $P_{0}$, due to the fact the performance may be limited by the other parameters, such as the processing time and the flying speed of the UAV. The 'Equal Time Allocation' and 'Semi-circle Trajectory' schemes are far worse than the other schemes especially in the case of 4 UEs, for not being able to make good use of the energy.

Results in Fig. 10 examines the convergence of the proposed algorithm by presenting the WSCTB results for four situations. The results clearly show that the proposed algorithm can converge to proper solutions in all the situations after 3 (for case of 4 UEs) or 4 (for case of 8UEs) iterations. Besides, the heterogeneity of UEs' weights can help to improve WSCTB since higher weights can attract UAV to provide higher level of services. Combining the results with that in Figs. 7-9, we can observe that the performance of WSCTB in a larger serving area (case of $8 \mathrm{UEs}$ ) is worse than that serving a smaller area (case of 4 UEs), and this is quite reasonable since not only the harvested energy of UAV and UEs decrease but also the consumed energy of the UAV and UEs increase in a larger serving area, leading to the shrinking of WSCTBs.

Table II presents the running time of the proposed Algorithm 1, which corresponds to a computer with 64-bit Intel(R) Core(TM) i5-9600KF CPU @3.7GHz and 16 GB RAM, running Matlab 2018a on . From the obtained data, we can observe that the algorithm running time 
increases with the number of time slots $N$ and the number of UEs $K$, which coincides with the intuition that the complexity of the algorithm increases with the two parameters.

TABLE II

ALGORITHM RUNNING TIME

\begin{tabular}{|c|c|c|c|c|c|c|c|c|}
\hline$(\mathrm{N}, \mathrm{K})$ & $(50,4)$ & $(60,4)$ & $(70,4)$ & $(80,4)$ & $(50,8)$ & $(60,8)$ & $(70,8)$ & $(80,8)$ \\
\hline Running Time (s) & 135.32 & 154.91 & 181.14 & 217.49 & 244.51 & 274.66 & 342.24 & 434.81 \\
\hline
\end{tabular}

\section{CONCLUSION}

A wireless-powered MEC setup exploiting cooperation between the AP and the UAV has been investigated, where the UAV serves as a moving MEC server as well as an energy and information relay. The WSCTB of the UEs was maximized under both the information causality and energy harvesting causality constraints, by jointly optimizing the computation task allocation, time allocation and the UAV's energy transmit power and trajectory. We proposed a three-step block coordinate descending algorithm to solve the formulated non-convex optimization problem. The UAV's trajectories under different settings were studied in the simulation results, verifying the key roles of the AP's location and the UEs' weight values. Results also illustrated that significant performance improvement can be achieved using the proposed algorithm.

In our future work, it would be interesting to explore the UAV-assisted MEC architecture in a long-term setting, and a larger serving area with multiple APs, UAVs. In this case, the air-toground channel model considering the LoS and NLoS probabilities [35] should be utilized to feature the more practical time-varying environment. The optimization of adjusting the UAVs' flying altitudes, the cooperation strategy between the UAVs, and UAVs' handover among cells should also be addressed accordingly. In addition, learning-based methods will be designed to decrease the algorithm complexity while maintaining a satisfactory performance.

\section{APPENDiX A: PROOF OF THEOREM 1}

The partial Lagrange function of problem (P1) is given as

$$
\begin{aligned}
& \mathcal{L}_{1}\left(\mathbf{L}, \mathbf{P}_{\mathrm{U}}, \boldsymbol{\gamma}, \boldsymbol{\mu}, \boldsymbol{\xi}, \boldsymbol{\rho}, \boldsymbol{\lambda}, \boldsymbol{\nu}\right)=\sum_{k=1}^{K}\left\{w_{k}\left(\sum_{n=1}^{N} L_{k}^{\mathrm{loc}}[n]+\sum_{n=1}^{N-2} L_{k}^{\mathrm{off}}[n]\right)\right. \\
& +\sum_{n=1}^{N-2} \widehat{\gamma}_{k, n} L_{k}^{\mathrm{off}}[n]-\sum_{n=2}^{N-1} \widetilde{\gamma}_{k, n}\left(L_{\mathrm{U}, k}[n]+L_{\mathrm{U}, k}^{\mathrm{off}}[n]\right)+O_{k} \sum_{n=2}^{N-1} \widehat{\mu}_{k, n}\left(L_{\mathrm{U}, k}[n]+L_{\mathrm{U}, k}^{\mathrm{off}}[n]\right)-\sum_{n=3}^{N} \widetilde{\mu}_{k, n} L_{\mathrm{U}, k}^{\text {down }}[n] \\
& +\lambda_{k}\left(\sum_{n=1}^{N-2} L_{k}^{\text {off }}[n]-\sum_{n=2}^{N-1}\left(L_{\mathrm{U}, k}[n]+L_{\mathrm{U}, k}^{\mathrm{off}}[n]\right)\right)+\nu_{k}\left(\sum_{n=3}^{N} L_{\mathrm{U}, k}^{\mathrm{down}}[n]-O_{k} \sum_{n=2}^{N-1}\left(L_{\mathrm{U}, k}[n]+L_{\mathrm{U}, k}^{\text {off }}[n]\right)\right) \\
& \left.+\sum_{n=1}^{N} \widetilde{\xi}_{k, n}\left(\eta_{k} \tau h_{k}[n] P_{\mathrm{U}}[n]-E_{k}[n]\left(\mathbf{L}, \mathbf{t}_{\chi}, \mathbf{u}_{\chi}\right)\right)\right\}+\sum_{n=1}^{N} \widetilde{\rho}_{n}\left(\eta_{\mathrm{U}} \tau h_{\mathrm{AP}}[n] P_{0}-E_{\mathrm{U}}[n]\left(\mathbf{L}, \mathbf{P}_{\mathrm{U}}, \mathbf{t}_{\chi}, \mathbf{u}_{\chi}\right)\right),
\end{aligned}
$$


where $\gamma, \boldsymbol{\mu}, \boldsymbol{\xi}, \boldsymbol{\rho}, \boldsymbol{\lambda}, \boldsymbol{\nu}$ respectively denote the sets of $\gamma_{k, n}, \mu_{k, n}, \xi_{k, n}, \rho_{n}, \lambda_{k}, \nu_{k}$ for $k \in \mathcal{K}, n \in \mathcal{N}$. Besides, $\widetilde{\gamma}_{k, n}=\sum_{i=n}^{N-1} \gamma_{k, i}, \widehat{\gamma}_{k, n}=\sum_{i=n+1}^{N-1} \gamma_{k, i}, \widetilde{\mu}_{k, n}=\sum_{i=n}^{N} \mu_{k, i}, \widehat{\mu}_{k, n}=\sum_{i=n+1}^{N} \mu_{k, i}, \widetilde{\xi}_{k, n}=$ $\sum_{i=n}^{N} \xi_{k, i}, \widetilde{\rho}_{n}=\sum_{i=n}^{N} \rho_{i}$. The task allocation parameters in $\overline{\mathbf{L}}_{\chi+1}$ shown in Theorem 1 can be obtained by applying the Karush-Kuhn-Tucker (KKT) conditions [33] and setting the derivatives of $\mathcal{L}_{1}\left(\mathbf{L}, \mathbf{P}_{\mathrm{U}}, \boldsymbol{\gamma}, \boldsymbol{\mu}, \boldsymbol{\xi}, \boldsymbol{\rho}, \boldsymbol{\lambda}, \boldsymbol{\nu}\right)$ w.r.t. $L_{k}^{\text {loc }}[n], L_{k}^{\text {off }}[n], L_{\mathrm{U}, k}[n], L_{\mathrm{U}, k}^{\text {off }}[n], L_{\mathrm{U}, k}^{\text {down }}[n]$ to zero.

With the obtained computation task allocation $\overline{\mathbf{L}}_{\chi+1}$, next, we will show how to obtain the optimal solution of the UAV's energy transmit power $\mathbf{P}_{\mathrm{U}, \chi+1}$. It is clear that a lower bound of $P_{\mathrm{U}}[n]$ can be obtained from the constraints in (26h), i.e.,

$$
P_{\mathrm{U}}[n] \geq \max _{k \in \mathcal{K}}\left\{\frac{\sum_{i=1}^{n} E_{k}[i]\left(\overline{\mathbf{L}}_{\chi+1}, \mathbf{t}_{\chi}, \mathbf{u}_{\chi}\right)}{\eta_{k} \tau h_{k, \chi}[n]}-\frac{\sum_{i=1}^{n-1} h_{k, \chi}[i] P_{\mathrm{U}}[i]}{h_{k, \chi}[n]}\right\} \triangleq P_{\mathrm{U}}^{\text {low }}[n] .
$$

Also, an upper bound of $P_{\mathrm{U}}[n]$ can be obtained from (26i) as

$$
P_{\mathrm{U}}[n] \leq \frac{1}{\tau} \sum_{i=1}^{n}\left[\eta_{\mathrm{U}} \tau P_{0} h_{\mathrm{AP}, \chi}[i]-E_{\mathrm{U}}^{\mathrm{prop}}[i]-\sum_{k=1}^{K}\left(E_{\mathrm{U}, k}[i]+E_{\mathrm{U}, k}^{\mathrm{off}}[i]+E_{\mathrm{U}, k}^{\mathrm{down}}[i]\right)\right]-\sum_{i=1}^{n-1} P_{\mathrm{U}}[i] \triangleq P_{\mathrm{U}}^{\mathrm{up}}[n],
$$

The first derivative of $\mathcal{L}_{1}$ w.r.t. $P_{\mathrm{U}}[n]$ is given by

$$
\frac{\partial \mathcal{L}_{1}}{\partial P_{\mathrm{U}}[n]}=\tau\left(\sum_{k=1}^{K} \eta_{k} h_{k, \chi}[n] \widetilde{\xi}_{k, n}^{*}-\widetilde{\rho}_{n}^{*}\right), \forall n
$$

If $\frac{\partial \mathcal{L}_{1}}{\partial P_{\mathrm{U}}[n]}>0$, i.e., $\widetilde{\rho}_{n}^{*}<\sum_{k=1}^{K} \eta_{k} h_{k, \chi}[n] \widetilde{\xi}_{k, n}^{*}$, the optimal $P_{\mathrm{U}}[n]$ should be its maximum, i.e., $P_{\mathrm{U}}^{\mathrm{up}}[n]$; otherwise if $\frac{\partial \mathcal{L}_{1}}{\partial P_{\mathrm{U}}[n]} \leq 0$, the optimal $P_{\mathrm{U}}[n]$ should be its minimum, i.e., $P_{\mathrm{U}}^{\text {low }}[n]$, as shown in (33) of Theorem 1.

If the given dual variables are optimal, denoted as $\gamma^{*}, \mu^{*}, \xi^{*}, \rho^{*}, \lambda^{*}$ and $\nu^{*}$, then the corresponding solution in Theorem 1 is also optimal, i.e., $\overline{\mathbf{L}}_{\chi+1}$ and $\mathbf{P}_{\mathrm{U}, \chi+1}$.

\section{APPENDIX B: PROOF OF LEMMA 2}

With the obtained Lagrange multipliers related to the inequality constraints, i.e., $\gamma_{j+1}, \boldsymbol{\mu}_{j+1}$, $\boldsymbol{\xi}_{j+1}, \boldsymbol{\rho}_{j+1}$ in Lemma 1, the Lagrange multipliers $\boldsymbol{\lambda}_{j+1}$ and $\boldsymbol{\nu}_{j+1}$ related to the equality constraints can be achieved through a bi-section search. According to the equality constraints in (26f) and (26g), the value of $\sum_{n=1}^{N-2} \bar{L}_{k, j+1}^{\text {off }}[n]$ can be described in three forms as in (B.1)-(B.3) with the expressions of the optimal solution given in Theorem 1.

$$
\begin{aligned}
\sum_{n=1}^{N-2} \bar{L}_{k, j+1}^{\text {off } *}[n] & =B \sum_{n=1}^{N-2} t_{k, \chi}^{\text {off }}[n]\left[\log _{2}\left[B h_{k, \chi}[n]\left(w_{k}+\lambda_{k, j+1}+\widehat{\gamma}_{k, n, j+1}\right)\right]^{+}-\log _{2}\left(N_{0} \ln 2 \widetilde{\xi}_{k, n, j+1}\right)\right]^{+} \\
& =\frac{B}{O_{k}} \sum_{n=3}^{N} t_{\mathrm{U}, k, \chi}^{\text {down }}[n]\left[\log _{2}\left[B h_{k, \chi}[n]\left(\nu_{k, j+1}-\widetilde{\mu}_{k, n, j+1}\right)\right]^{+}-\log _{2}\left(N_{0} \ln 2 \widetilde{\rho}_{n, j+1}\right)\right]^{+} \\
& =\sum_{n=2}^{N-1}\left\{t_{k, \chi}[n] \sqrt{\frac{1}{3 \kappa_{\mathrm{U}} C_{k}^{3} \widetilde{\rho}_{n, j+1}}\left[O_{k} \widehat{\mu}_{k, n, j+1}-\widetilde{\gamma}_{k, n, j+1}-O_{k} \nu_{k, j+1}-\lambda_{k, j+1}\right]^{+}}+B t_{\mathrm{U}, k, \chi}^{\text {off }}[n] \times\right.
\end{aligned}
$$




$$
\left.\left[\log _{2}\left[B h_{\mathrm{AP}, \chi}[n]\left(O_{k} \widehat{\mu}_{k, n, j+1}-\widetilde{\gamma}_{k, n, j+1}-O_{k} \nu_{k, j+1}-\lambda_{k, j+1}\right)\right]^{+}-\log _{2}\left(N_{0} \ln 2 \widetilde{\rho}_{n, j+1}\right)\right]^{+}\right\},
$$

The expression (B.1) is obtained from the expression of $\bar{L}_{k, j+1}^{\mathrm{off}}[n]$, (B.2) is derived from the equation $\sum_{n=1}^{N-2} \bar{L}_{k, j+1}^{\text {off }}[n]=\frac{1}{O_{k}} \sum_{n=3}^{N} \bar{L}_{\mathrm{U}, k, j+1}^{\text {down }}[n]$ between (26f) and (26g), and (B.3) comes from the right side of (26f).

From equations (B.1) and (B.2), we can see that both $\lambda_{k, j+1}$ and $\nu_{k, j+1}$ are monotonic nondecreasing implicit functions of $\sum_{n=1}^{N-2} \bar{L}_{k, j+1}^{\text {off }}[n]$, and thus the function expressed in (B.3) is a monotonic non-increasing function of $\sum_{n=1}^{N-2} \bar{L}_{k, j+1}^{\mathrm{off}}[n]$. Given the value of $\sum_{n=1}^{N-2} \bar{L}_{k, j+1}^{\mathrm{off}}[n]$, the corresponding $\lambda_{k, j+1}$ and $\nu_{k, j+1}$ can be obtained through the bi-section search method within the ranges of $\lambda_{k, j+1} \in\left[\lambda_{k, j+1}^{\min }, \lambda_{k, j+1}^{\max }\right]$ and $\nu_{k, j+1} \in\left[\nu_{k, j+1}^{\min }, \nu_{k, j+1}^{\max }\right]$. The obtained $\lambda_{k, j+1}$ and $\nu_{k, j+1}$ should make sure that the right side values of (B.1) and (B.2), denoted as $V_{k, j+1}^{\mathrm{C} 1}$ and $V_{k, j+1}^{\mathrm{C} 2}$, satisfy that $\sum_{n=1}^{N-2} \bar{L}_{k, j+1}^{\mathrm{off}}[n]=V_{k, j+1}^{\mathrm{C} 1}\left(\lambda_{k, j+1}\right)=V_{k, j+1}^{\mathrm{C} 2}\left(\nu_{k, j+1}\right)$. The expressions of $\lambda_{k, j+1}^{\min }$, $\lambda_{k, j+1}^{\max }, \nu_{k, j+1}^{\min }, \nu_{k, j+1}^{\max }$ given in Lemma 2 are obtained from (B.1)-(B.3) considering the monotonic decreasing property of $\widehat{\gamma}_{k, n, j+1}, \widetilde{\gamma}_{k, n, j+1}, \widetilde{\mu}_{k, n, j+1}, \widehat{\mu}_{k, n, j+1}$ w.r.t. the slot index $n$.

\section{REFERENCES}

[1] X. Hu, K. Wong, and Z. Zheng, "Wireless-powered mobile edge computing with cooperated UAV," in proc. Int. Workshop Signal Process. Adv. Wireless Commun. (SPAWC), Cannes, France, Jul. 2019, pp. 1-5.

[2] D. Sabella, A. Vaillant, P. Kuure, U. Rauschenbach, and F. Giust, "Mobile-edge computing architecture: The role of mec in the internet of things," IEEE Cons. Elec. Mag., vol. 5, no. 4, pp. 84-91, Oct. 2016.

[3] Y. Mao, C. You, J. Zhang, K. Huang, and K. B. Letaief, "A survey on mobile edge computing: The communication perspective," IEEE Commun. Surveys Tuts., vol. 19, no. 4, pp. 2322-2358, fourthquarter 2017.

[4] P. Mach and Z. Becvar, "Mobile edge computing: A survey on architecture and computation offloading," IEEE Commun. Surveys Tuts., vol. 19, no. 3, pp. 1628-1656, thirdquarter 2017.

[5] T. Q. Dinh, J. Tang, Q. D. La, and T. Q. S. Quek, "Offloading in mobile edge computing: Task allocation and computational frequency scaling," IEEE Trans. Commun., vol. 65, no. 8, pp. 3571-3584, Aug. 2017.

[6] C. You, K. Huang, H. Chae, and B. H. Kim, "Energy-efficient resource allocation for mobile-edge computation offloading," IEEE Trans. Wireless Commun., vol. 16, no. 3, pp. 1397-1411, Mar. 2017.

[7] J. Zhang, X. Hu, Z. Ning, E. C. . Ngai, L. Zhou, J. Wei, J. Cheng, and B. Hu, "Energy-latency tradeoff for energy-aware offloading in mobile edge computing networks," IEEE Internet Things J., vol. 5, no. 4, pp. 2633-2645, Aug. 2018.

[8] T. X. Tran and D. Pompili, "Joint task offloading and resource allocation for multi-server mobile-edge computing networks," IEEE Trans. Veh. Technol., vol. 68, no. 1, pp. 856-868, Jan. 2019.

[9] X. Hu, L. Wang, K. Wong, M. Tao, Y. Zhang, and Z. Zheng, "Edge and central cloud computing: A perfect pairing for high energy efficiency and low-latency," IEEE Trans. Wireless Commun., pp. 1-1, 2019.

[10] M. Liu, F. R. Yu, Y. Teng, V. C. M. Leung, and M. Song, "Distributed resource allocation in blockchain-based video streaming systems with mobile edge computing," IEEE Trans. Wireless Commun., vol. 18, no. 1, pp. 695-708, Jan. 2019. 
[11] C. You, K. Huang, and H. Chae, "Energy efficient mobile cloud computing powered by wireless energy transfer," IEEE J. Sel. Areas Commun., vol. 34, no. 5, pp. 1757-1771, May 2016.

[12] X. Hu, K. Wong, and K. Yang, "Wireless powered cooperation-assisted mobile edge computing," IEEE Trans. Wireless Commun., vol. 17, no. 4, pp. 2375-2388, Apr. 2018.

[13] F. Wang, J. Xu, X. Wang, and S. Cui, "Joint offloading and computing optimization in wireless powered mobile-edge computing systems,” IEEE Trans. Wireless Commun., vol. 17, no. 3, pp. 1784-1797, Mar. 2018.

[14] Y. Mao, J. Zhang, and K. B. Letaief, "Dynamic computation offloading for mobile-edge computing with energy harvesting devices," IEEE J. Sel. Areas Commun., vol. 34, no. 12, pp. 3590-3605, Dec. 2016.

[15] Y. Zeng, R. Zhang, and T. J. Lim, "Wireless communications with unmanned aerial vehicles: Opportunities and challenges," IEEE Commun. Mag., vol. 54, no. 5, pp. 36-42, May 2016.

[16] N. Cheng, W. Xu, W. Shi, Y. Zhou, N. Lu, H. Zhou, and X. Shen, "Air-ground integrated mobile edge networks: Architecture, challenges, and opportunities," IEEE Commun. Mag., vol. 56, no. 8, pp. 26-32, Aug. 2018.

[17] F. Zhou, R. Q. Hu, Z. Li, and Y. Wang, "Mobile edge computing in unmanned aerial vehicle networks," arXiv preprint arXiv:1910.10523, 2019.

[18] S. Jeong, O. Simeone, and J. Kang, "Mobile edge computing via a UAV-mounted cloudlet: Optimization of bit allocation and path planning," IEEE Trans. Veh. Technol., vol. 67, no. 3, pp. 2049-2063, Mar. 2018.

[19] F. Cheng, S. Zhang, Z. Li, Y. Chen, N. Zhao, F. R. Yu, and V. C. M. Leung, "UAV trajectory optimization for data offloading at the edge of multiple cells," IEEE Trans. Veh. Technol., vol. 67, no. 7, pp. 6732-6736, Jul. 2018.

[20] X. Zhang, Y. Zhong, P. Liu, F. Zhou, and Y. Wang, "Resource allocation for a UAV-enabled mobile-edge computing system: Computation efficiency maximization,” IEEE Access, vol. 7, pp. 113 345-113 354, 2019.

[21] X. Hu, K. Wong, K. Yang, and Z. Zheng, "UAV-assisted relaying and edge computing: Scheduling and trajectory optimization," IEEE Trans. Wireless Commun., vol. 18, no. 10, pp. 4738-4752, Oct. 2019.

[22] F. Zhou, Y. Wu, R. Q. Hu, and Y. Qian, "Computation rate maximization in UAV-enabled wireless-powered mobile-edge computing systems," IEEE J. Sel. Areas Commun., vol. 36, no. 9, pp. 1927-1941, Sep. 2018.

[23] F. Zhou, Y. Wu, H. Sun, and Z. Chu, "UAV-enabled mobile edge computing: Offloading optimization and trajectory design," in proc. IEEE Inter. Conf. Commun. (ICC), Kansas City, MO, USA, May 2018.

[24] Y. Huo, X. Dong, T. Lu, W. Xu, and M. Yuen, "Distributed and multilayer UAV networks for next-generation wireless communication and power transfer: A feasibility study," IEEE Internet Things J., vol. 6, no. 4, pp. 7103-7115, Aug 2019.

[25] J. Ouyang, Y. Che, J. Xu, and K. Wu, "Throughput maximization for laser-powered uav wireless communication systems," in proc. IEEE Inter. Conf. Commun. Workshops (ICC Workshops), Kansas City, USA, May 2018, pp. 1-6.

[26] D. Killinger, "Free space optics for laser communication through the air," Optics and Photonics News, vol. 13, no. 10, pp. 36-42, 2002.

[27] T. J. Nugent and J. T. Kare, "Laser power for UAVs," LaserMotive White Paper C Power Beaming for UAVs, 2010.

[28] M. Grant, S. Boyd, and Y. Ye, “CVX: Matlab software for disciplined convex programming," 2008.

[29] Y. Zeng and R. Zhang, "Energy-efficient UAV communication with trajectory optimization," IEEE Trans. Wireless Commun, vol. 16, no. 6, pp. 3747-3760, Jun. 2017.

[30] LTE Unmanned Aircraft Systems-Trial Report, Qualcomm Technol., Inc., San Diego, CA, USA, May 2017.

[31] W. Zhang, Y. Wen, K. Guan, D. Kilper, H. Luo, and D. O. Wu, "Energy-optimal mobile cloud computing under stochastic wireless channel," IEEE Trans. Wireless Commun., vol. 12, no. 9, pp. 4569-4581, Sep. 2013. 
[32] Y. Zeng, Q. Wu, and R. Zhang, "Accessing from the sky: A tutorial on UAV communications for 5G and beyond," arXiv preprint arXiv:1903.05289, 2019.

[33] S. Boyd and L. Vandenberghe, Convex optimization. Cambridge university press, 2004.

[34] D. P. Bertsekas and J. N. Tsitsiklis, Parallel and distributed computation: Numerical methods. Prentice hall Englewood Cliffs, NJ, 1989, vol. 23.

[35] A. Al-Hourani, S. Kandeepan, and S. Lardner, "Optimal lap altitude for maximum coverage," IEEE Wireless Communications Letters, vol. 3, no. 6, pp. 569-572, Dec 2014. 